\title{
Synopsis of the terrestrial and freshwater gastropod fauna of southern Bahia, Brazil
}

\author{
Fernanda dos Santos Silva ${ }^{1,3}$; Luiz R.L. Simone ${ }^{1,4}$ \& Rodrigo Brincalepe Salvador ${ }^{2,5}$ \\ 1 Universidade de São Paulo (USP), Museu de Zoologia (MZUSP). São Paulo, SP, Brasil. \\ ${ }^{2}$ Museum of New Zealand Te Papa Tongarewa. Wellington, New Zealand. \\ ${ }^{3}$ ORCID: http://orcid.org/0000-0002-2213-0135. E-mail: fernanda06@usp.br (corresponding author) \\ ${ }^{4}$ ORCID: http://orcid.org/0000-0002-1397-9823. E-mail: Irsimone@usp.br \\ ${ }^{5}$ ORCID: http://orcid.org/0000-0002-4238-2276. E-mail: salvador.rodrigo.b@gmail.com
}

\begin{abstract}
The terrestrial and freshwater malacofauna of southern Bahia is little known, especially in comparison to the well-studied eastern portion of the state covered by remnants of Atlantic Forest. We present here a synopsis of all gastropod species known from the central southern region of Bahia state, known as 'Centro-Sul Baiano', focusing on four municipalities: Condeúba, Cordeiros, Mortugaba, and Piripá. The list herein contains data from the literature, historical material deposited in museum collections, and two recent expeditions undertaken in the region. The survey resulted in 21 species of gastropods, with nearly 700 voucher specimens (mostly freshwater) deposited in the collection of the Museu de Zoologia da Universidade de São Paulo (São Paulo, Brazil). Circa 30\% of the species are non-indigenous; previously, the only known exotic in the region was Melanoides tuberculata (Müller, 1774). The family Achatinidae was the most diverse group in number of native species, while the typically diverse superfamily Orthalicoidea was represented by only two species.
\end{abstract}

Keywords. Caatinga; Cerrado; Gastropoda; Mollusca; Taxonomy.

\begin{abstract}
Resumo. Sinopse da fauna gastrópode terrestre e de água doce do sul da Bahia, Brasil. A malacofauna terrestre e de água doce do sul da Bahia é pouco conhecida, principalmente em comparação com a bem estudada porção leste do estado coberta por remanescentes de Mata Atlântica. Apresentamos aqui uma sinopse de todas as espécies de gastrópodes conhecidas da região centro-sul do estado da Bahia, conhecido como 'Centro-Sul Baiano', com foco em quatro municípios: Condeúba, Cordeiros, Mortugaba e Piripá. A lista aqui apresentada contém dados da literatura, material histórico depositado em coleções de museus e duas expedições recentes realizadas na região. 0 levantamento resultou em 21 espécies de gastrópodes, com cerca de 700 espécimes (principalmente de água doce) depositados na coleção do Museu de Zoologia da Universidade de São Paulo (São Paulo, Brasil). Cerca de 30\% das espécies são não indígenas; anteriormente, o único exótico conhecido na região era Melanoides tuberculata (Müller, 1774). A família Achatinidae foi o grupo mais diverso em número de espécies nativas, enquanto a superfamília tipicamente diversa Orthalicoidea foi representada por apenas duas espécies.
\end{abstract}

Palavras-Chave. Caatinga; Cerrado; Gastropoda; Mollusca; Taxonomia.

\section{INTRODUCTION}

The central southern region of Bahia state in Brazil (the 'Centro-Sul Baiano'; IBGE, 1990) is predominantly covered by the hypoxerophylic Caatinga, which is a diverse but still little-known biome (Silva et al., 2003). Recent papers (e.g., Salvador \& Cavallari, 2012, 2014; Simone \& Casati, 2013; Porto et al., 2016; Salvador \& Simone, 2016; Salvador, 2018; Silva et al., 2019a; Simone \& Salvador, 2021) have demonstrated a surprising number of new records and new taxa of mollusks in this region.
However, knowledge of the local malacofauna is still incipient and needs special attention, not only because it is possibly an area of high potential endemism (Cavallari et al., 2016; Salvador, 2019), but also because non-marine gastropods have the highest extinction rates among invertebrates (Lydeard et al., 2004; Régnier et al., 2009). Thus, we present herein a synopsis of all known species of terrestrial and freshwater gastropods from the central southern portion of Bahia state based on literature records, museum collections, and new collection efforts, the latter focusing on the municipalities of Condeúba, Cordeiros, Mortugaba and Piripá. 


\section{MATERIAL AND METHODS}

Specimens sampling were carried out in two instances: December/2017 to January/2018 (rainy season) and August/2018 (dry season). The expedition focused on four municipalities (Condeúba, Cordeiros, Mortugaba, and Piripá; Fig. 1), which are located on a trasitional zone of mixed Cerrado and Caatinga biome features (Oliveira et al., 2017). Given the paucity of records for the region, the sampling points were arbitrarily chosen (both in rural and urban areas) (Fig. 2), giving preference to areas where snails were more likely to be present, estimated from literature data and personal experience. Collection effort consisted of 10 minutes in each point (roughly $1 \mathrm{~m}^{2}$ ) and included visual search (dislodging substrate and litter), and sampling of leaf litter in a subset of the points. Voucher specimens were deposited in the malacological collection of the Museu de Zoologia da Universidade de São Paulo (MZSP, São Paulo, Brazil).

Additional records informations were gathered from the literature and specimens in the collection of the MZSP. Species identification was based on original descriptions, published catalogues (Simone, 2006; Birckolz et al., 2016) and further taxonomic literature, as well as comparative specimens (including type material when necessary). The species are listed below following the arrangement of Bouchet et al. (2017), including data on their type localities, geographic distribution, a list of analized material, and, when appropriate, further remarks.

Measurements were taken with a digital caliper (for larger specimens) and under stereomicroscope coupled with computer-assisted camera and software (Zeiss Axiovision SE64 Rel 4.8).

Abbreviations used herein as follows: col. = collector $(\mathrm{s})$; colln. = collection; $\mathrm{sh}=$ empty shell(s); spm = whole specimen(s); $\mathrm{H}=$ shell height; $\mathrm{D}=$ shell greater diameter.

Given that the taxonomy of Sarasinula Grimpe \& Hoffmann, 1924 and Megalobulimus Miller, 1878 still need revisionary work, we have sequenced the barcoding region of the mitochondrial COI marker (circa 650 bp) of our specimens for future reference. Selected adult specimens of these genera had a small section of their foot clipped for DNA extraction, which was achieved with DNeasy ${ }^{\circledR}$ Blood \& Tissue Kit (QIAGEN N.V.), following standard protocol. The primers used were LCOI and HCOI (Folmer et al., 1994). PCR protocol as follows: (1) initial denaturation, at $96^{\circ} \mathrm{C}$ ( 2 minutes); (2) 35 cycles of: (a) denaturation, at $95^{\circ} \mathrm{C}$ (30 seconds); (b) annealing, at $48^{\circ} \mathrm{C}$ (1 minute); (c) extension, at $72^{\circ} \mathrm{C}$ (2 minutes); (3) final extension, at $72^{\circ} \mathrm{C}$ (5 minutes). The PCR products were quantified via agarose gel electrophoresis, then cleaned following standard ExoSAP-IT ${ }^{\mathrm{TM}}$ protocol (Affymetrix Inc.), and finally Sanger sequenced. The resulting sequences

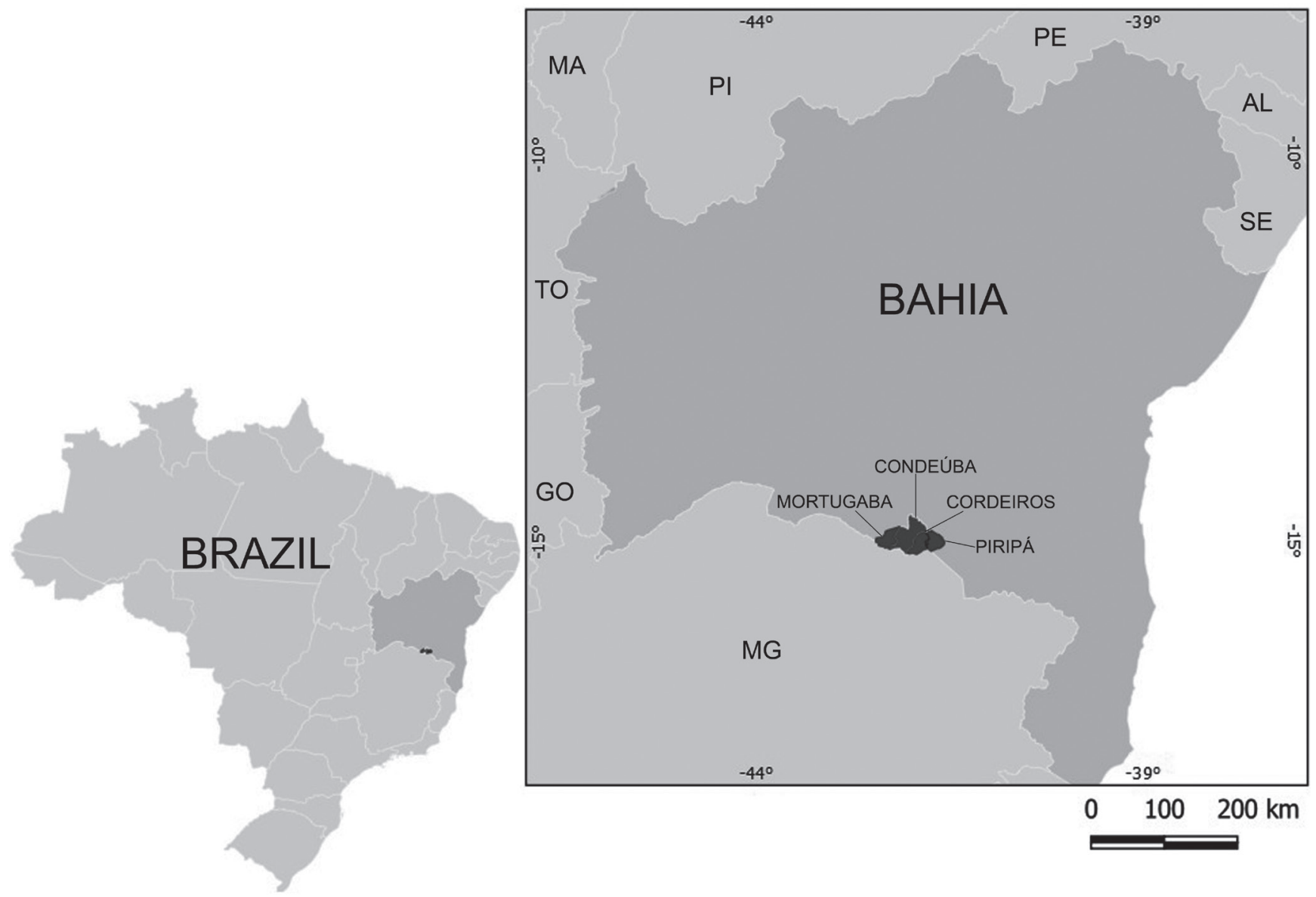

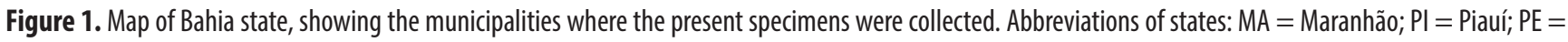
Pernambuco; $\mathrm{AL}=$ Alagoas; $\mathrm{SE}=$ Sergipe; $\mathrm{TO}=$ Tocantins; $\mathrm{GO}=\mathrm{Goiás} ; \mathrm{MG}=$ Minas Gerais. 


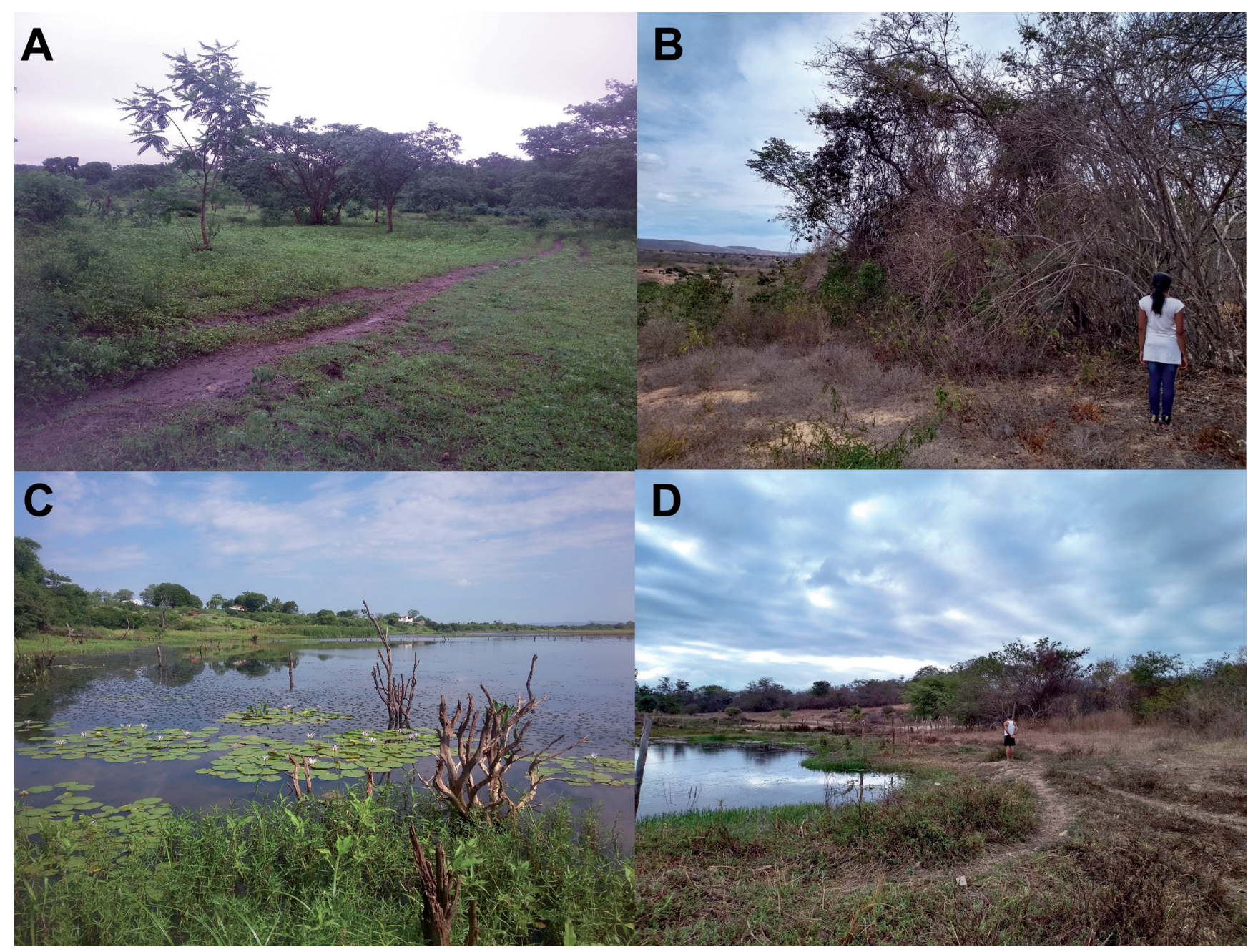

Figure 2. Examples of locations where the 2017-2018 collections took place. (A) Mortugaba municipality, rural area, January/2018. (B) Mortugaba municipality, rural area, August/2018. (C) Cordeiros municipality, dam, January/2018. (D) Cordeiros municipality, dam, August/2018.

were assembled, and quality proofed using the software Geneious Prime (v. 2019.0.3, Biomatters Ltd.). The consensus sequences were then uploaded to NCBI GenBank; register numbers are presented below, under the entries of Sarasinula and Megalobulimus species.

\section{RESULTS \\ Systematics \\ Caenogastropoda Superfamily Ampullarioidea \\ Family Ampullariidae \\ Genus Pomacea Perry, 1810 Pomacea canaliculata (Lamarck, 1822) Figs. 3A-B}

Synonymy, see Scott (1957: 299); Castellanos \& Fernandez (1976: 13); Simone (2004: 398). Complement:

Ampullaria canaliculata: Bertoni, 1925: 72; Pitoni, 1984: 247; Darrigran, 1991: 213; Cuezzo \& Drahg, 1995: 199.

Pomacea canaliculata: Solem, 1956: 2; Scott, 1957: 299; Lopes, 1957: 43; Shade, 1965: 219; Klappenbach,
1967: 42; Castellanos \& Fernandez, 1976: 13; Thiengo et al., 1998: 233; Cowie et al., 2001: 13; Cowie \& Thiengo, 2003: 58; Simone, 2004: 398, figs. 11-12, 88-89, 120-121, 199-205, 2006: 49, fig. 69; Thiengo et al., 2005: 869; Estebenet et al., 2006: 329; Gregoric et al., 2006: 53; Agudo-Padrón, 2008: 151, 2014: 10; Martello et al., 2008: 33; Agudo-Padrón \& Lenhard, 2011: 165, figs. 3-6; Hayes et al., 2012: 737, figs. 1, 4-8, 10, 11, 13, 14; Madella \& Auricchio, 2014: 55; Birckolz et al., 2016: 148.

Pomacea (Pomacea) canaliculata canaliculata: Quintana, 1982: 109.

\section{Type locality: Guadeloupe.}

Occurrence: Guyana, Trinidad, Bolivia, Brazil (Amazonas, Pará, Rondônia, Mato Grosso, Goiás, Mato Grosso do Sul, Bahia, Pernambuco, Rio de Janeiro, Rio Grande do Sul and Santa Catarina states), Paraguay, Uruguay and Argentina (Simone, 2006; Birckolz et al., 2016).

Material examined: BRAZIL: Bahia; Condeúba, $14^{\circ} 54^{\prime} 02.39^{\prime \prime} S$, 42 $02^{\prime} 18.92^{\prime \prime} \mathrm{W}, \mathrm{MZSP} 136686$, 1 sh (F.S. Silva col. 10/i/2018); MZSP 136654, 47 sh (F.S. Silva col. 10/i/2018); MZSP 139790, 7 sh (F.S. Silva col. 05/viii/2018); Piripá, $15^{\circ} 02^{\prime} 28.09^{\prime \prime} \mathrm{S}, 41^{\circ} 42^{\prime} 17.78^{\prime \prime} \mathrm{W}, \mathrm{MZSP} 136684,40$ sh 
(F.S. Silva col. 09/i/2018); MZSP 136681, 15 sh (2017); MZSP 139669, 3 sh (F.S. Silva col. 06/viii/2018).

Remarks: This species is native to Central and South America but is invasive in several parts of the world. It is unknown whether the species is native to Bahia or if it has been introduced there. Only empty shells were collected from dry areas and standing waters of the Gavião River and weirs in Piripá and Condeúba municipalites.

\section{Genus Asolene d'Orbigny, 1838 \\ Asolene meta (Ihering, 1915) \\ Figs. 3C-D}

Ampullaria meta lhering, 1915: 12, figs. 6-7; Cowie \& Thiengo, 2003: 69.

Ampullarius (Ampullarius) meta: Morretes, 1949: 67.

Pomacea meta: Simone, 2006: 55, fig. 92.

Asolene meta: Soares et al., 2006: 107.

Type locality: São Franscisco River, Barra municipality, Bahia state, Brazil.

Occurrence: Known only from type locality (Simone, 2006).

Material examined: BRAZIL: Bahia; Guanambi, $14^{\circ} 13^{\prime} 09.86^{\prime \prime} \mathrm{S}, 42^{\circ} 49^{\prime} 46.82^{\prime \prime} \mathrm{W}$, MZSP 26689, 2 sh (Giannotti col. 15/vii/1981).

Remarks: This species was only represented by historical specimens (from 1981) in the MZSP collection, which represents an expansion of range about $350 \mathrm{~km}$ southward. Since the species could not be found in our renewed collection efforts, it is unknown if it still inhabits the area.

\section{Superfamily Cerithioidea Family Thiaridae \\ Genus Melanoides Oliver, 1804 \\ Melanoides tuberculata (Müller, 1774) Figs. 3E-F}

Synonymy, see Starmühler (1969: 224); Brandt (1974: 164); and Simone (2001: 156). Complement:

Nerita tuberculata Müller, 1774: 191.

Melania tuberculata: Carvalho, 1986: 57; Souza et al., 1998: 451.

Melanoides tuberculata: Oliveira et al., 1981: 97; Freitas et al., 1987:302;Silvaetal., 1994:439; Pazetal., 1995:79;Thiengo et al., 1998: 233; Martins-Silva \& Barros, 2001: 867; França et al., 2007: 42; Massemin et al., 2009: 366; Strong et al., 2011: 54; Kotzian \& Amaral, 2013: 302; Agudo-Padrón, 2014: 11; Paula et al., 2017: 829.

Melanoides tuberculatus: Fernandez et al., 2001: 280; Simone, 2001: 156, figs. 9, 10, 46, 47, 54, 55, 89, 169-188; Fernandez et al., 2003: 78; Vidigal et al., 2005: 69; RochaMiranda \& Martins-Silva, 2006: 1109; Agudo-Padrón, 2008: 152, 2011:56; Santos \& Eskinazi-Sant'Anna, 2010: 1, fig. 2; Santos et al., 2010: 511; Silva \& Gomes, 2014: 145.
Type locality: Coromandel Coast, India.

Occurrence: Naturally distributed in Asia and eastern Africa; introduced to the Americas and Polynesia (RochaMiranda \& Martins-Silva, 2006).

Material examined: BRAZIL: Bahia; Andaraí, 12³9.54'32"S, 4112.01'76"W, MZSP 121907, 25 sh (C.E.F. Rocha col. 14/

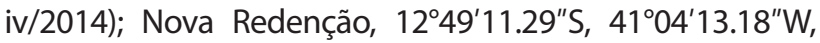
MZSP 106217, 12 sh (DEBE-UFSCar col. 18/ix/2004); MZSP 135902, 6 sh (M.E. Bichuette col. 01/v/2004); MZSP 137069, 6 sh (M.E. Bichuette col. 01/v/2004); Jussiape, $13^{\circ} 31^{\prime} 00.57^{\prime \prime} \mathrm{S}, 41^{\circ} 35^{\prime} 31.73^{\prime \prime} \mathrm{W}, \mathrm{MZSP} 78758,23$ sh (L.S. Rocha col. 19/xii/1998); Condeúba, 1454'02.39"S, 4202'18.92"W, MZSP 136589, 28 sh (F.S. Silva col. 10/i/2018); MZSP 138591, 33 sh (F.S. Silva col. 10/i/2018); Piripá, $15^{\circ} 02^{\prime} 28.09^{\prime \prime}$, 4142'17.78"W, MZSP 136587, 154 sh (F.S. Silva col. 09/i/2018); MZSP 136588, 172 sh (F.S. Silva col. 07/i/2018).

Remarks: Despite being a common and widespread invasive species, only empty shells were found in the new sampling efforts. The specimens were retrieved from dry ground and standing waters of Gavião River and weirs in Condeúba and Piripá municipalites.

\section{Família Hemisinidae \\ Gênero Aylacostoma Spix, 1827 Aylacostoma sp. Figs. 3G-H}

Material examined: BRAZIL: Bahia; Condeúba, $14^{\circ} 54^{\prime} 02.39^{\prime \prime} \mathrm{S}, 42^{\circ} 02^{\prime} 18.92^{\prime \prime} \mathrm{W}, \mathrm{MZSP} 136593,1$ sh (F.S. Silva col. 10/i/2018).

Remarks: A single deteriorated specimen was found in a weir in Condeúba municipality. Its last whorl was broken and the periostracum eroded, precluding identification to species level.

\section{Heterobranchia \\ Superfamily Lymnaeoidea \\ Family Physidae \\ Genus Stenophysa Martens, 1898 \\ Stenophysa marmorata (Guilding, 1828) \\ Figs. 3I-J}

Synonymy, see Quintana (1982: 120). Complement:

Physa marmorata Guilding, 1828: 534; Tillier, 1980: 38;

Gómez et al., 1986: 131; Paraense, 1986: 459, figs. 1-33;

Gnaspini \& Trajano, 1994: 550; Lima, 1995a: 85, fig. 5;

Thiengo et al., 1998: 233.

Aplexa rivalis: Beck, 1837: 116.

Physa rivalis: Gray, 1854: 25.

Physa Braziliensis: Clessin, 1888: 170.

Physa (Physella?) marmorata: Baker, 1930: 42.

Aplexa marmorata: Clench, 1936: 337; Aguayo, 1938: 213;

Leme, 1966: 269; Oliveira et al., 1981: 307; Cuezzo \&

Drahg, 1995: 199; Agudo-Padrón, 2008: 155, 2014: 13. 
Aplexa (Stenophysa) marmorata: Quintana, 1982: 120; Simone, 2006: 101, fig. 301; Agudo-Padrón \& Lenhard, 2011: 166.

Stenophysa marmorata: Coimbra-Jr. \& Santos, 1986: 231; Darrigran, 1991: 213; Taylor, 2003: 113, figs. 95-108, pl. 5, fig. 3; Gregoric et al., 2006: 53; Martello et al., 2008: 33; Núñes, 2011: 98. Díaz \& Martín, 2013: 26; Trouy et al., 2016: 121.
Type locality: Saint Vincent, Lesser Antilles.

Occurrence: Central and South America (Simone, 2006).

Material examined: BRAZIL: Bahia; Condeúba, $14^{\circ} 54^{\prime} 02.39^{\prime \prime} \mathrm{S}, 42^{\circ} 02^{\prime} 18.92^{\prime \prime} \mathrm{W}, \mathrm{MZSP} 139670$, 1 sh (F.S. Silva col. 05/viii/18). Cordeiros, $15^{\circ} 02^{\prime} 23,90^{\prime \prime} \mathrm{S}$, $41^{\circ} 56^{\prime} 02,07^{\prime \prime}$ W, MZSP 136604, 2 sh (F.S. Silva col. 02/i/18);
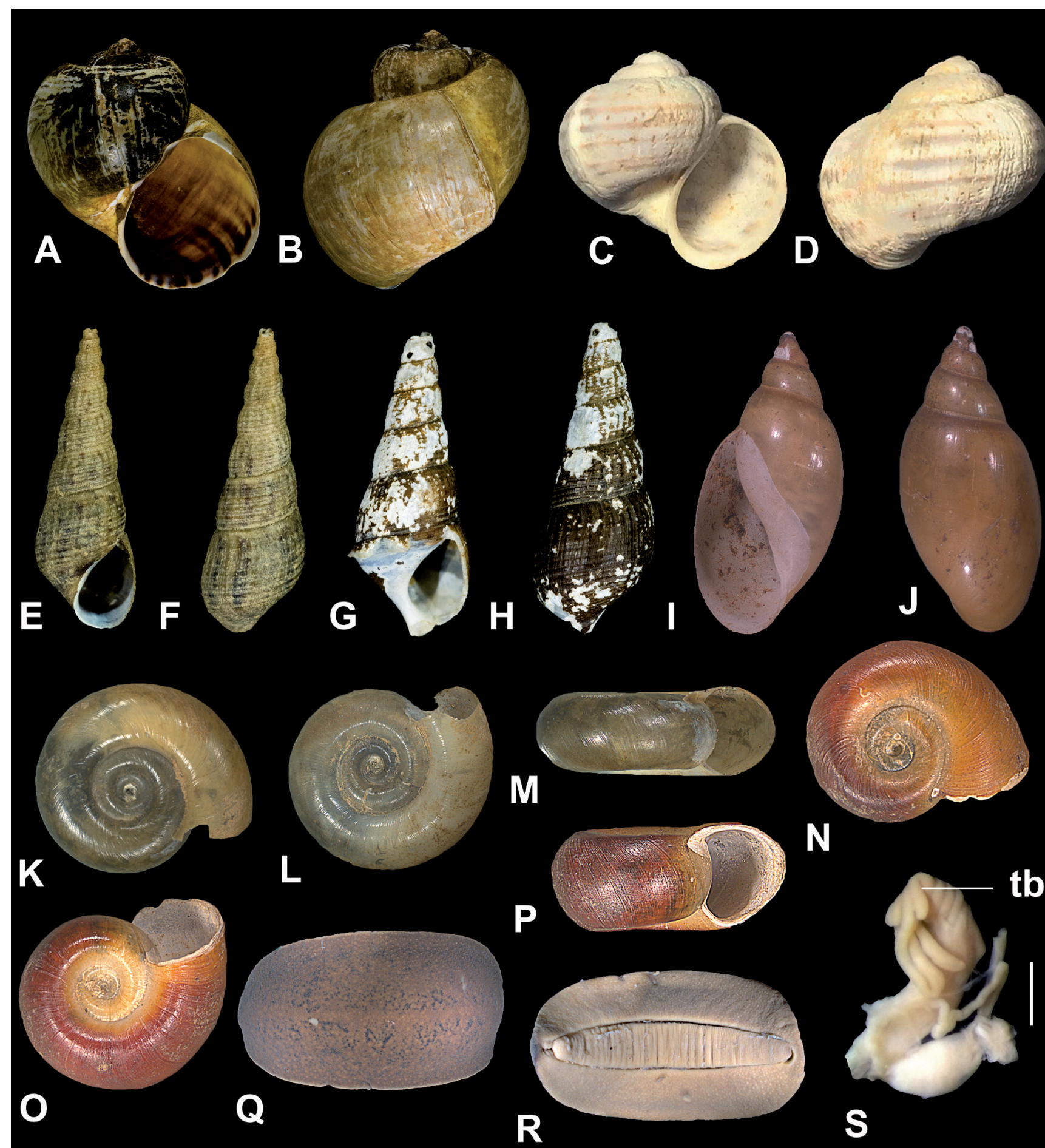

Figure 3. Shells from Bahia state. (A-B) Pomacea canaliculata, MZSP 136681, Piripá (H=90,1 mm). (C-D) Asolene meta, MZSP 26689, Guanambi (H=30.7 mm). (E-F) Melanoides tuberculata, MZSP 136589, Condeúba (H=17.9 mm). (G-H) Aylacostoma sp., MZSP 136593, Condeúba (H=26.7 mm). (I-J) Stenophysa marmorata, MZSP 139671, Piripá ( $H=15.0 \mathrm{~mm}$ ). (K-M) Biomphalaria glabrata, MZSP 136662, Piripá (D=9.93 mm). (N-P) Biomphalaria straminea, MZSP 136659, Cordeiros ( $(\mathrm{D}=9.25 \mathrm{~mm}$ ). (Q-R) Sarasinula plebeia, MZSP 136616, Cordeiros ( $\mathrm{H}=36.7 \mathrm{~mm})$. (S) Penial gland of Sarasinula plebeia (Scale bar: $2 \mathrm{~mm}$; tb = tubules). 
MZSP 139664, 7 sh (F.S. Silva col. 13/viii/18); Piripá, $15^{\circ} 02^{\prime} 28.09^{\prime \prime} \mathrm{S}, 41^{\circ} 42^{\prime} 17.78^{\prime \prime} \mathrm{W}, \mathrm{MZSP} 139671$, 1 sh (F.S. Silva col. 06/viii/18).

\section{Family Planorbidae \\ Genus Biomphalaria Preston, 1910 Biomphalaria glabrata (Say, 1818) Figs. 3K-M}

Synonymy, see Arias (1952: 59). Complement:

Planorbis glabratus Say, 1818: 280.

Planorbis olivaceus Spix, 1827: 26, pl. 18, fig. 2; Lutz, 1918: 50.

Planorbis ferrugineus Spix, 1827: 26, pl. 18, fig. 1; d'Orbigny, 1835a: 544, 1835b: 26; Gray, 1854: 25; Lutz, 1918: 50.

Planorbis guadeloupensis Sowerby, 1820: 20; Martins, 1938: 33; Jay, 1839; Martens, 1873: 195; Lutz, 1918: 52; Baker, 1913: 661; Ernest, 1948: 79; Jaeckel, 1952: 6.

Australorbis olivaceus: Morretes, 1953: 60.

Planorbis bahiensis: Martens, 1868: 187.

Helisoma (Planorbina) guadeloupensis guadeloupensis: Baker, 1930: 43.

Helisoma (Planorbina) olivacea: Haas, 1939: 266.

Australorbis glabratus Pilsbry, 1934; 43; Martins, 1938: 28; Baker, 1945a: 90, pl. 45, fig. 1; Morretes, 1949: 125; Barbosa \& Dobbin-Jr., 1951: 2; Arias, 1952: 59; Morretes, 1953: 59; Olivier \& Barbosa, 1955a: 79; Paraense \& Deslandes, 1955: 87; Paraense, 1958: 66; Kloetzel, 1959: 280; Magalhães, 1964: 283.

Australorbis glabratus olivaceus: Lucena, 1951: 94, 1953: 246, 1956: 64.

Biomphalaria glabrata: Basch, 1968: 21; Barbosa \& Figueiredo, 1969: 286; Sodeman-Jr., 1973: 103; Michelson \& DuBois, 1974: 102; Paraense, 1975: 115, figs. 45-48, 2006: 21; Boffi, 1979: 30; Pieri et al., 1980: 83; Tillier, 1980: 39, pl. 3, fig. 2; Luz et al., 1981: 107; Freitas et al., 1987: 299; Rosa, 1987: 311; Figueiredo, 1989: 383; Barbosa, 1992: 311; Silva et al., 1994: 439; Lima, 1995b: 94, fig. 24; Souza et al., 1996: 542; Carvalho et al., 1998: 39; Luz et al., 1998: 41; Thiengo et al., 1998: 233; Souza et al., 1998: 451; Oliveira \& Almeida, 1999: 14; Fernandez et al., 2001: 280; Souza et al., 2001: 294; Pointier et al., 2005: 250; Simone, 2006: 105, fig. 313; Agudo-Padrón, 2008: 155, 2014: 13; Massemin et al., 2009: 370; Pepe et al., 2009: 783; Carvalho et al., 2018: 3.

Type locality: Guadeloupe.

Occurrence: Central and South America (Simone, 2006).

Material examined: BRAZIL: Bahia; Andaraí,

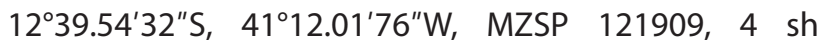
(14/iv/2014); Maracás, 13³1'50.64"S, 40³3'12.24"W, MZSP 31599, 13 sh (W. Bokerman col. xi/1965); Vitória da Conquista, $14^{\circ} 51^{\prime} 42.93^{\prime \prime} \mathrm{S}, 40^{\circ} 50^{\prime} 40.32^{\prime \prime} \mathrm{W}$, MZSP 98332, 6 sh (ex J. Vaz colln.); Condeúba, 1454'02.39"S, 4202'18.92"W,MZSP 136693, 1 sh (F.S.Silva col. 10/i/2018);
MZSP 139668, 2 sh (F.S. Silva col. 05/viii/2018); Cordeiros, $15^{\circ} 02^{\prime} 23,90^{\prime \prime} \mathrm{S}, 41^{\circ} 56^{\prime} 02,07^{\prime \prime} \mathrm{W}, \mathrm{MZSP} 139666,2$ sh (F.S. Silva col. 04/viii/2018); MZSP 139667, 4 sh (F.S. Silva col. 13/viii/2018); Piripá, $15^{\circ} 02^{\prime} 28.09^{\prime \prime} \mathrm{S}, 41^{\circ} 42^{\prime} 17.78^{\prime \prime} \mathrm{W}$, MZSP 136662, 1 sh (F.S. Silva col. 09/i/2018).

Remarks: Biomphalaria glabrata is a widespread neotropical freswater species known as intermediary host of schistosomiasis and can often be found in anthropically disturbed areas (Massemin et al., 2009). It had already been reported from other 23 municipalites in the central southern region of Bahia (Carvalho et al., 2018); the new occurrences complement that list.

\section{Biomphalaria straminea (Dunker, 1848) Figs. 3N-P}

Synonymy, see Lucena (1956: 40). Complement:

Planorbis stramineus Dunker, 1848: 42; Baker, 1913: 662; Lutz, 1918: 60; Ernest, 1948: 79.

Planorbis centimetralis Lutz, 1918: 53, pl. 7, figs. 8a-d; Martins, 1938: 34.

Tropicorbis centimetralis Lutz, 1918: 52; Morretes, 1949: 125; Lucena, 1953: 246; Olivier \& Barbosa, 1955b: 105.

Planorbina (Tropicorbis) straminea: Baker, 1930: 46.

Helisoma (Tropicorbis) centimetralis: Haas, 1939: 267.

Tropicorbis stramineus: Lucena, 1951: 95, 1953: 246, 1956: 40; Morretes, 1953: 59.

Biomphalaria straminea: Barbosa \& Figueiredo, 1969: 286; Paraense, 1975:118,figs.54-55,2006:21;Boffi, 1979:30; Tillier, 1980: 40, pl. 3, fig. 3; Oliveira et al., 1981: 313; Rosa, 1987: 311; Teles \& Vaz, 1988: 173; Figueiredo, 1989: 383; Teles et al., 1991: 350; Barbosa, 1992: 311; Ferrari \& Hofmann, 1992: 33; Lima, 1995b: 101, fig. 27; Souza et al., 1996: 542; Teles, 1996: 342; Carvalho et al., 1998: 39; Luz et al., 1998: 41; Souza et al., 1998: 451; Thiengo et al., 1998: 233; Oliveira \& Almeida, 1999: 14; Fernandez et al., 2001: 280; Agudo-Padrón, 2008: 156, 2014: 14; Martins-Silva \& Barros, 2001: 867; Souza et al., 2001: 294; Pointier et al., 2005: 250; Simone, 2006: 109, fig. 321; Pepe et al., 2009: 783; Kotzian \& Amaral, 2013: 306; Díaz \& Martín, 2013: 26; Carvalho et al., 2018: 3.

Type locality: South America, restricted by Martens (1873) to Lagunilha and Caracas, Venezuela (see also Paraense, 1975).

Occurrence: Central America, Guyana, Venezuela and Brazil (Simone, 2006).

Material examined: BRAZIL: Bahia; Guanambi, $14^{\circ} 13^{\prime} 09.86^{\prime \prime} \mathrm{S}, 42^{\circ} 49^{\prime} 46.82^{\prime \prime} \mathrm{W}, \mathrm{MZSP} 31549$, 3 sh (F. Gianmots col. 15/xiii/1991); Condeúba, 1454'02.39"S, 4202'18.92"W, MZSP 140927, 1 sh (F.S. Silva col. 05/ viii/2018); Cordeiros, $15^{\circ} 02^{\prime} 23,90^{\prime \prime} \mathrm{S}$, $41^{\circ} 56^{\prime} 02,07^{\prime \prime} \mathrm{W}$, MZSP 136659, 34 sh (F.S. Silva col. 02/i/2018); MZSP 139665, 3 sh (F.S. Silva col. 13/viii/2018); Piripá, 
$15^{\circ} 02^{\prime} 28.09^{\prime \prime} \mathrm{S}, 41^{\circ} 42^{\prime} 17.78^{\prime \prime} \mathrm{W}, \mathrm{MZSP} 136663,3$ sh (F.S. Silva col. 09/i/2018).

Remarks: This species has a sympatric occurrence with B. glabrata, present in regions with longer dry seasons and being more resistant to dessication than its congener (Lucena, 1951). It is also an intermediary host of schistosomiasis and has been reported from 12 municipalites of the central southern region of Bahia (Carvalho et al., 2018); the new occurrences complement that list.

\section{Superfamília Veronicelloidea Família Veronicellidae Gênero Sarasinula Grimpe \& Hoffmann, 1924 Sarasinula plebeia (Fischer, 1868)} Figs. 3Q-S

Synonymy, see Gomes \&Thomé (2004:591). Complement: Vaginula behni: Morretes, 1949: 128; Thomé, 1969: 103. Vaginulus plebeius: Thomé, 1975: 158.

Sarasinula plebeia: Andrews \& Dundee, 1987: 165; Thomé, 1988: 809, 1993: 67; Mansur \& Thomé, 1994: 41; Thomé, 1994:45; Thomé et al., 1997:530; Thomé et al., 1999: 161; Rueda et al., 2002: 115; Maceira, 2003: 459; Simone, 2006: 97, fig. 275; Thomé et al., 2006: 55; Naranjo-Garcia et al., 2007: 45; Agudo-Padrón, 2008: 153, 2014: 11.

Type locality: New Caledonia.

Occurrence: USA, Mexico, Central America, Antilles, Colombia, Venezuela, Brazil (Para to Rio Grande do Sul) and Chile (Simone, 2006).

Material examined: BRAZIL: Bahia; Cordeiros, $15^{\circ} 02^{\prime} 23.90^{\prime \prime} \mathrm{S}, 41^{\circ} 56^{\prime} 02.07^{\prime \prime} \mathrm{W}, \mathrm{MZSP} 136616,5$ spm (F.S. Silva col. 04/i/2018).

Barcoding: GenBank reg nr MN792580 (MZSP 136616).

Remarks: Identification of Sarasinula spp. is not trivial and the taxonomy of the genus is still in flux. The anatomical characters observed in our specimens are in line with previous studies on S. plebeia, mainly due to the short club-like penis with 6-8 short tubules (Fig. 3S; Hoffmann, 1925). Furthermore, our COI sequence displayed $99 \%$ identity with those of voucher specimens of S. plebeia stemming from other regions in South America available in GenBank (from Gomes et al., 2013, unpublished).

\section{Superfamily Achatinoidea Family Achatinidae Genus Lissachatina Bequaert, 1950 Lissachatina fulica (Bowdich, 1822)} Figs. 4A-B

Synonymy, see Miquel \& Herrera (2014: 120). Complement:

Helix fulica Férussac, 1821: 49.
Achatina fulica Bowdich, 1822: pl. 8; Oliveira et al., 1981: 329; Teles et al., 1997: 311; Oliveira \& Almeida, 1999: 25; Vasconcellos \& Pile, 2001: 582; Kosloski \& Fischer, 2002: 66; Raut \& Baker, 2002: 55; Teles \& Fontes, 2002: 3; Fischer \& Colley, 2004: 44, 2005: 128; Eston et al., 2006: 173; Fischer et al., 2006: 1; Simone, 2006: 308, fig. 10; Thomé et al., 2006: 69; Thiengo et al., 2007; Graeff-Teixeira, 2007: 743; Teixeira et al., 2008: 334; Albuquerque et al., 2008: 837, 2009: 880; Santos et al., 2010: 513; Silva \& Aleluia, 2010: 199; Zanol et al., 2010: 448; Vogler et al., 2013: 39; Silva \& Omena, 2014: 2; Madella \& Auricchio, 2014: 53; SáOliveira et al., 2016: 160; Valim \& Bim, 2017: 7.

Achatina (Lissachatina) fulica Bequaert, 1950: 54; AgudoPadrón, 2011: 56.

Lissachatina fulica: Miquel \& Herrera, 2014: 120, fig. 36.

Type locality: Africa.

Occurrence: Natural from Africa, but introduced in the Americas, Southeast Asia and Oceania (Zanol et al., 2010).

Material examined: BRAZIL: Bahia; Cordeiros, $15^{\circ} 02^{\prime} 23.90^{\prime \prime} \mathrm{S}, 41^{\circ} 56^{\prime} 02.07^{\prime \prime} \mathrm{W}, \mathrm{MZSP} 136582$, 1 sh (F.S. Silva col. 03/i/2018).

Remarks: The giant African land snail is presently distributed in 25 (out of 26) Brazilian states (Zanol et al., 2010; Darrigran et al., 2020); the exception being the typically undersampled state of Acre (Salvador et al., 2020; Lima et al., 2021). Despite being common in several regions of Brazil, only one specimen (an empty shell) was recovered in the present study.

\section{Genus Beckianum Baker, 1961 Beckianum beckianum (Pfeiffer, 1846) Figs. 4C-D}

Bulimus beckianus Pfeiffer, 1846: 82.

Synopeas (Opeas) beckiana: Dall, 1896: 415.

Synopeas beckianum: Richards \& Hummelinck, 1940: 9; Baker, 1945b: 91; Altena, 1960: 50; Baker, 1961: 84.

Opeas beckianum: Baker, 1913: 644; Pilsbry, 1906: 189, pl. 27, figs. 42-46, 54-55, 1910: 502; Haas, 1939: 268; Schalie, 1940: 5; Morretes, 1949: 132; Jaeckel, 1952: 6; Ramírez et al., 2003: 276; Salgado \& Coelho, 2003: 155; Santos et al., 2010: 514.

Opeas (Synopeas) beckianum: Baker, 1927: 7; Pilsbry, 1930: 342.

Opeas (Opeas) beckianum: Haas, 1953, 205.

Beckianum beckianum: Marcus \& Marcus, 1968: 204; Santos \& Monteiro, 2001: 186; Simone, 2006: 187, fig. 685; Massemin et al., 2009: 414, pl. 9E; Robinson et al., 2009: 632, fig. 7A; Almeida \& Mota, 2011: 75; Kotzian \& Amaral, 2013: 306; Simone \& Casati, 2013: 156; Salvador \& Simone, 2015: 3, fig. 9; Salvador et al., 2015: 75, fig. 30; Birckolz et al., 2016: 150, table 1; Breure et al., 2016: 35, fig. 19; Salvador et al., 2018: 114, figs. 9K-M; Lima et al., 2021: 273, figs. 3Q-S. 
Beckianum beckianum beckianum: Thompson, 2011: 523.

Type locality: Opara Island, Polynesia (Pfeiffer, 1846). Pilsbry (1906) considered this mistaken, arguing that Pfeiffer's specimens were most similar to those found in Central America (see also Salvador \& Simone, 2015).

Occurrence: Mexico to Brazil (Pará, Fernando de Noronha, Bahia, Rio Grande do Norte, Rondônia, Rio de Janeiro, and São Paulo states), including the Caribbean Islands (Simone, 2006; Birckolz et al., 2016).

Material examined: BRAZIL: Bahia; Condeúba, $14^{\circ} 54^{\prime} 02.39^{\prime \prime} \mathrm{S}, 4^{\circ} 02^{\prime} 18.92^{\prime \prime} \mathrm{W}, \mathrm{MZSP}$ 139788, 1 sh (F.S. Silva col. 04/viii/2018); Cordeiros, $15^{\circ} 02^{\prime} 23,90^{\prime \prime}$, 41 ${ }^{\circ} 56^{\prime} 02,07^{\prime \prime}$ W, MZSP 139784, 4 spm (F.S. Silva col. 04/viii/2018); Piripá, $15^{\circ} 02^{\prime} 28.09^{\prime \prime} \mathrm{S}, \quad 41^{\circ} 42^{\prime} 17.78^{\prime \prime} \mathrm{W}$, MZSP 139785, 6 sh (F.S. Silva col. 07/viii/2018).

Remarks: Live specimens were only found during the dry season.

\section{Genus Lamellaxis Strebel \& Pfeiffer, 1882 Lamelaxis gracilis (Hutton, 1834)} Figs. 4E-F

Synonymy, see Silva et al. (2019b: 3). Complement: Achatina subula Pfeiffer, 1839: 352.

Opeas gracile: Schalie, 1940: 5.

Lamelaxis gracilis: Karlin, 1956: 122; Scarabino, 2003: 207; Santos et al., 2010: 514; Simone \& Casati, 2013: 156; González-Valdivia et al., 2018: 7; Lim et al., 2018: 114; Silva et al., 2019b: 3, fig. 2H-I.

Lamelaxis (Allopeas) gracilis: Coomans, 1967: 135.

Allopeas gracilis: Robinson et al., 2009: 632, fig. 7B; Thompson, 2011: 159; Miquel \& Jaime, 2018: 5.

Allopeas gracile: Delannoye et al., 2015: 258, pl. 51; Breure et al., 2016: 33, fig. 15; Capinera, 2017: 116; D'Ávila et al., 2018: 1553.

Type locality: Mirzapur, India.

Occurrence: West Indies to South America; introduced to tropical and subtropical areas in Asia, Australia and Polynesia (Simone, 2006; Capinera, 2017).

Material examined: BRAZIL: Bahia; Condeúba, $14^{\circ} 54^{\prime} 02.39^{\prime \prime} \mathrm{S}, 42^{\circ} 02^{\prime} 18.92^{\prime \prime} \mathrm{W}, \mathrm{MZSP}$ 136658, 2 sh (F.S. Silva col. 11/i/2018); MZSP 138549, 3 sh (F.S. Silva col. 04/i/2018); Mortugaba, $15^{\circ} 01^{\prime} 42,20^{\prime \prime} \mathrm{S}, 42^{\circ} 22^{\prime} 30.79^{\prime \prime} \mathrm{W}$, MZSP 136655, 4 sh (F.S. Silva col. 03/i/2018); Cordeiros, $15^{\circ} 02^{\prime} 23,90^{\prime \prime} \mathrm{S}, 41^{\circ} 56^{\prime} 02,07^{\prime \prime} \mathrm{W}, \mathrm{MZSP} 136657,1 \mathrm{sh}$ (F.S. Silva col. 28/xii/2017); Piripá, $15^{\circ} 02^{\prime 2} 28.09^{\prime \prime} S$,

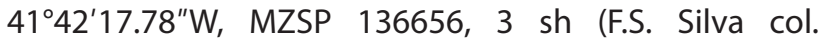
08/i/2018).

Remarks: Generally found in dry environments (Delannoye et al., 2015). Present specimens were all colleted in urban areas.

\section{Genus Leptinaria Beck, 1837 \\ Leptinaria unilamellata (d'Orbigny, 1838) Figs. 4G-H}

Synonymy, see Silva et al. (2019b: 3). Complement: Achatina lamellata Potiez \& Michaud, 1838: 128, pl. 11, figs. 7-8; Reeve, 1849a: pl. 18, fig. 97.

Leptinaria lamellata: Morretes, 1949: 132; Silva et al., 2019b: 3, figs. 2J-K; Salvador et al., 2021: table 2. Leptinaria (Leptinaria) lamellata: Coomans, 1967: 134. Leptinaria unilamellata: Robinson et al., 2009: 632, fig. 7B; Santos et al., 2010: 514; Breure et al., 2016: 35, fig. 14; D'Ávila et al., 2018: 1552. Miquel \& Jaime, 2018: 5; Breure et al., 2020: 23, fig. 5; Lima et al., 2021: 274, figs. 3T-V.

Type locality: Undefined; nevertheless, Delannoye et al. (2015) quote Santa Cruz, Bolivia, as the possible type locality.

Occurrence: Nicaragua to Brazil (Simone, 2006; Delannoye et al., 2015).

Material examined: BRAZIL: Bahia, Condeúba, $14^{\circ} 54^{\prime} 02.39^{\prime \prime} \mathrm{S}, 42^{\circ} 02^{\prime} 18.92^{\prime \prime} \mathrm{W}, \mathrm{MZSP} 138552,1$ sh (F.S. Silva col., 11/i/2018).

Remarks: A single empty shell was found, collected in an urban area. This species is very common in anthropically disturbed environments, being likewise abundant in agricultural areas (Carvalho et al., 2009).

\section{Genus Opeas Beck, 1837 Opeas opella Pilsbry \& Vanatta, 1905 Figs. $41-J$}

Opeas opella Pilsbry \& Vanatta, 1905: 785, fig. 1; Pilsbry, 1906: 186, pl. 24, fig. 36; Baker, 1913: 645; Haas, 1939: 268; Morretes, 1949: 133; Marcus \& Marcus, 1968: 193; Cowie, 1998: 356; Salgado \& Coelho, 2003: 155; Simone, 2006: 309, fig. 13.

Type locality: Honolulu, Hawaii, EUA. Nevertheless, Cowie (1998) argued that the species should be native to Asia.

Occurrence: Natural distribuition include "East Indies" to China (Cowie, 2001).

Material examined: BRAZIL: Bahia; Cordeiros, $15^{\circ} 02^{\prime} 23.90^{\prime \prime} \mathrm{S}, 41^{\circ} 56^{\prime} 02.07^{\prime \prime} \mathrm{W}, \mathrm{MZSP} 138550,15 \mathrm{sh}$ (F.S. Silva col. 28/xii/2017); Mortugaba, $15^{\circ} 01^{\prime} 42.20^{\prime \prime}$, 42²2'30.79"W, MZSP 136653, 1 sh (F.S. Silva col. 03/i/2018).

Remarks: Only empty shells were found in our collection efforts. The species can be identified by its turriform shell, irregular sculpture and tall aperture. In Brazil, Opeas opella has been reported as an introduced species, 
but has been previously reported only from Pará state (Baker, 1913). The present new record from Bahia state represents a large extension in distribution in Brazil.

\section{Genus Stenogyra Shuttleworth, 1854 Stenogyra octogyra (Pfeiffer, 1856) Figs. 4K-L}

Bulimus octogyrus Pfeiffer, 1856: 45.
Opeas octogyra: Albers, 1860: 265.

Opeas octogyrum: Pilsbry, 1906: 206, pl. 29, figs. 75-79; Baker, 1913: 644; Haas, 1939: 268; Jaeckel, 1952: 6; Salgado \& Coelho, 2003: 155.

Obeliscus (Stenogyra) octogyrus: Baker, 1927: 4.

Obeliscus octogyrus: Pilsbry, 1933: 69.

Obeliscus (Stenogyra) octogyrus octogyrus: Morretes, 1949: 133.

Stenogyra octogyra: Simone, 2006: 188, fig. 695; Salvador et al., 2017: 140, fig. 17, 2018: 116, figs. 10I-J.

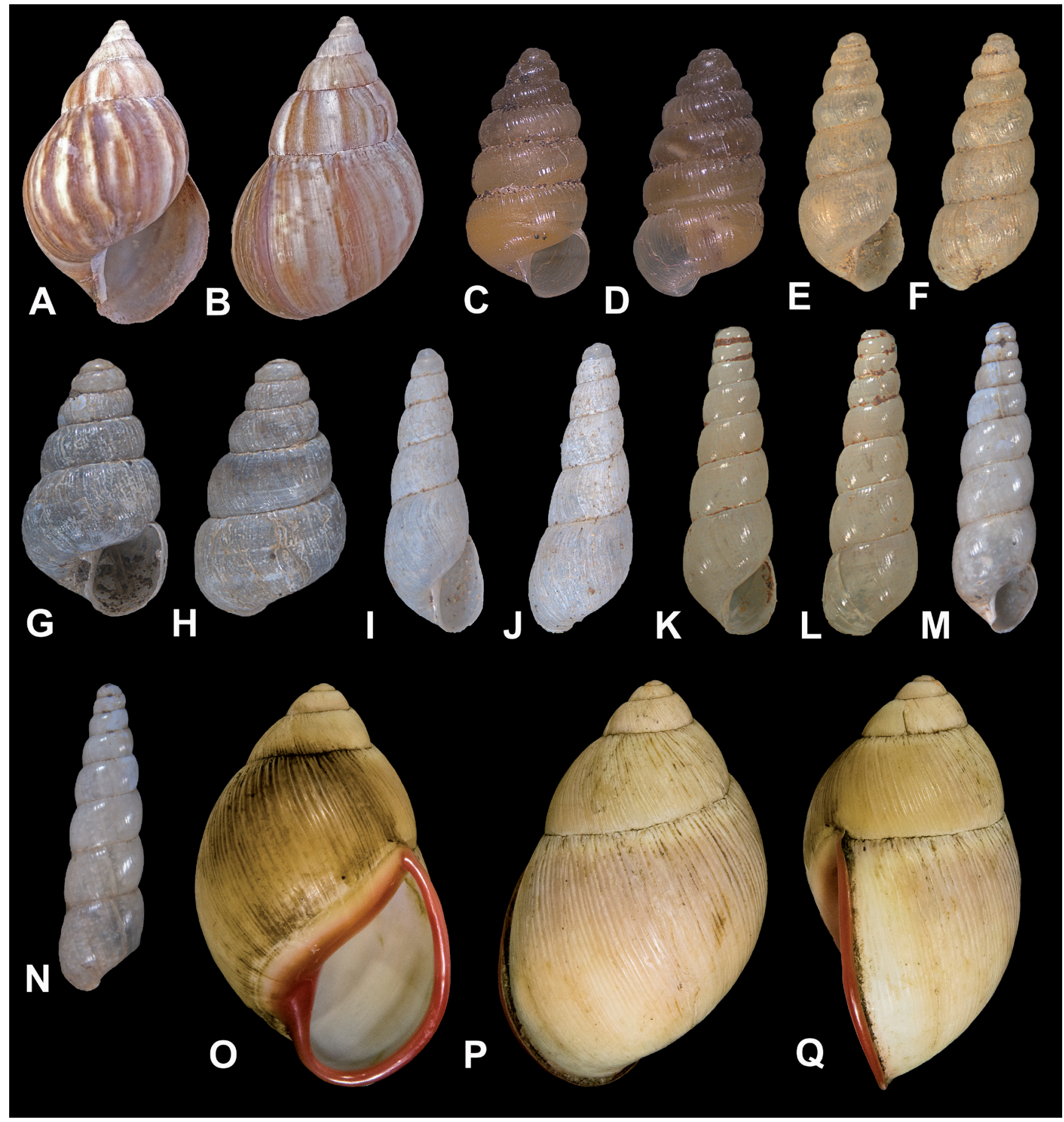

Figure 4. Shells from Bahia state. (A-B) Lissachatina fulica, MZSP 136582, Cordeiros (H =66.7 mm). (C-D) Beckianum beckianum, MZSP 139784, Cordeiros ( $\mathrm{H}=6.5 \mathrm{~mm})$. (E-F) Lamelaxis gracilis, MZSP 136658, Condeúba $(\mathrm{H}=6.4 \mathrm{~mm})$. (G-H) Leptinaria unilamellata, MZSP 138552, Condeúba (H=5.4 mm). (I-J) Opeas opella, MZSP 138550, Cordeiros (H=5.7 mm). (K-L) Stenogyra octogyra, MZSP 103040, Itaquara (H=10.7 mm). (M-N) Subulina octona, MZSP 136653, Mortugaba ( $\mathrm{H}=14.2 \mathrm{~mm})$. (0-0) Megalobulimus oblongus, MZSP 136679, Mortugaba $(H=117.5 \mathrm{~mm})$. 
Type locality: Caracas, Venezuela.

Occurrence: Venezuela to Brazil (Salvador et al., 2017).

Material examined: BRAZIL: Bahia; Andaraí, $12^{\circ} 39^{\prime} 54.32^{\prime \prime} \mathrm{S}, 41^{\circ} 12^{\prime} 01.76^{\prime \prime} \mathrm{W}, \mathrm{MZSP} 103030,2$ sh (30/ vii/2010); Itaquara, $13^{\circ} 27^{\prime} 13.47^{\prime \prime} \mathrm{S}, \quad 39^{\circ} 52^{\prime} 42.54^{\prime \prime} \mathrm{W}$, MZSP 103040, 2 sh (19/vi/2009).

Remarks: This species was not found during our collection efforts but is represented in the MZUSP collection. Even so, given that the species is widely distributed in South America, there is no reason to suspect it became locally extinct in southern Bahia, as it may be only rare in the region.

\section{Genus Subulina, Beck, 1837 \\ Subulina octona (Bruguière, 1792) Figs. 4M-N}

Synonymy, see Araújo \& Bessa (1993: 492). Complement: Bulimus octonus Bruguière, 1792: 325.

Helix octona: d'Orbigny, 1835b: 9; d'Orbigny, 1835a: 260. Achatina octona: Hidalgo, 1869: 138.

Rumina (Subulina) octona: Adams, 1866: 441.

Subulina octona: Pilsbry, 1906: 72, pl. 12, figs. 8-9; Baker, 1913: 609, 1927: 2, pl. 20, fig. 99; Haas, 1935: 111, 1939: 268; Richards \& Hummelinck, 1940: 13; Morretes, 1949: 131; Jaeckel, 1952: 6; Altena, 1960: 50; Coomans, 1967: 134; Marcus \& Marcus, 1968: 187; Götting, 1978: 105, fig. 6; Tillier, 1980: 58; Dutra, 1988: 582; Araújo \& Bessa, 1993: 492; Bessa \& Araújo, 1995: 719; Oliveira \& Almeida, 1999: 23; Santos \& Monteiro, 2001: 188; Salgado \& Coelho, 2003: 154; Simone, 2006: 308, fig. 11; Thomé et al., 2006: 70; Agudo-Padrón, 2008: 157; Massemin et al., 2009: 418, pl. 9B; Robinson et al., 2009: 633, fig. 7B; Santos et al., 2010: 514: Agudo-Padrón \& Lenhard, 2011: 169; Madella \& Auricchio, 2014: 55; D'Ávila et al., 2018: 1552; Miquel \& Jaime, 2018: 5; Lima et al., 2021: 274, figs. 4F-H.

Subulina (Subulina) octona: Haas, 1953: 253.

Subulina octonata: Oliveira et al., 1981: 327.

Type locality: Guadeloupe.

Occurrence: Naturally ocurring in tropical continental Americas, but introduced to the Caribbean Islands, Africa, Asia and Pacific Islands (Massemin et al., 2009; Miquel \& Herrera, 2014).

Material examined: BRAZIL: Bahia; Condeúba, $14^{\circ} 54^{\prime} 02.39^{\prime \prime} \mathrm{S}, 42^{\circ} 02^{\prime} 18.92^{\prime \prime} \mathrm{W}, \mathrm{MZSP} 138551,1 \mathrm{sh}$ (F.S. Silva col. 11/i/2018); Mortugaba, $15^{\circ} 01^{\prime} 42.20^{\prime \prime}$, $42^{\circ} 22^{\prime} 30.79^{\prime \prime} \mathrm{W}, \mathrm{MZSP} 136653,1$ sh (F.S. Silva col. 04/i/2018).

Remarks: The present specimens were all collected in urban areas.

\section{Superfamily Rhytidoidea \\ Family Strophocheilidae \\ Genus Megalobulimus Miller, 1878 \\ Megalobulimus oblongus (Müller, 1774) \\ Figs. 40-Q}

Synonymy, see Bequaert (1948: 58). Complement:

Bulimus oblongus: Hupé, 1857: 26; Ernest, 1948: 76.

Strophocheilus (Megalobulimus) oblongus: Bequaert, 1948: 58; Arias, 1952: 55, pl. 2, fig. 4, 1953: 249; Solem, 1956: 3; Sawaya \& Peterson, 1962: 33; Shade, 1965: 2015; Fernandez \& Castellanos, 1973: 276.

Strophocheilus (Megalobulimus) oblongus oblongus: Haas, 1959: 365.

Psiloicus oblongus Morretes, 1952: 113, 1953: 69; Oliveira et al., 1981: 340; Oliveira \& Almeida, 1999: 31.

Strophocheilus oblongus: Morretes, 1949: 142; Barattini \& Ledón, 1949: 2; Bigarella, 1951: 247; Jaeckel, 1952: 7; Lopes, 1957: 44; Fiori \& Jaeger, 1978: 847; Tillier, 1980: 57; Oliveira \& Oliveira, 1984: 8; Parkinson et al., 1987: pl. 15, fig. 1; Massemin et al., 2009: 425, pl. 7B.

Megalobulimus oblongus: Leme, 1975: 174; Götting, 1978: 105, fig. 8; Boffi, 1979: 32; Deisler \& Stange, 1982: 1; Vieira \& Simone, 1990: 57; Cuezzo \& Drahg, 1995: 196; Klappenbach, 1997: 45; Simone, 1999: 5, 2012: 438; Salgado \& Coelho, 2003: 158; AgudoPadrón, 2008: 161, 2011: 62, fig. 3; Santos et al., 2010: 513; Ramírez et al., 2012: 62, fig. 1G; Madella \& Auricchio, 2014: 57; Páez et al., 2014: 50, fig. 1; Roldán et al., 2014: 80; Breure \& Araújo, 2017: 105, fig. 38D; Gordillo, 2018: 64; Pilate et al., 2018: 74.

Megalobulimus oblongus oblongus: Quintana, 1982: 83.

Type locality: Undefined.

Occurrence: South America (Simone, 2006).

Material examined: BRAZIL: Bahia; Mortugaba, $15^{\circ} 01^{\prime} 42.20^{\prime \prime} \mathrm{S}, 42^{\circ} 22^{\prime} 30.79^{\prime \prime} \mathrm{W}, \mathrm{MZSP} 136652,2$ sh (F.S. Silva col. 03/i/2018); MZSP 136679, 1 spm (F.S. Silva col. 03/i/2018); MZSP 139663, 1 sh (F.S. Silva col. 11/viii/2018).

Barcoding: GenBank reg nr MN756630 (MZSP 136679).

Remarks: The specimens could be identified through conchological features (oblong-oval shell, blunt spire apex, and protoconch sculpture), as described and illustrated by Bequaert (1948) as the "typical M. oblongus". However, this species might represent a yet unresolved species complex.

\section{Megalobulimus conicus (Bequaert, 1948) Figs. 5A-C}

Strophocheilus oblongus conicus Bequaert, 1948: 79, pl. 6, fig. 1; pl. 19, fig. 2; pl. 23, figs. 1-3.

Strophocheilus (Strophocheilus) oblongus conicus: Haas, 1949: 152.

Psiloicus conicus: Morretes, 1952: 113, 1953: 69. 
Megalobulimus conicus: Salgado \& Coelho, 2003: 157; Simone, 2006: 208, fig. 791; Salvador et al., 2015: 75, figs. 28-29.

Type locality: Maranhão state, Brazil.

Occurrence: Brazil (Amazonas, Maranhão, Bahia, and Tocantins states) (Simone, 2006; Salvador et al., 2015).
Material examined: BRAZIL: Bahia; paratype MZSP 14610, 1 sh; Ibiajara, $12^{\circ} 59^{\prime} 00.88^{\prime \prime} S, 42^{\circ} 13^{\prime} 25.27^{\prime \prime} \mathrm{W}$, MZSP 29067, 1 sh (F.S. Silva col. 1986), Palmas de Monte Alto, $13^{\circ} 52^{\prime} 50.54^{\prime \prime} \mathrm{S}, 43^{\circ} 03^{\prime} 49.38^{\prime \prime} \mathrm{W}, \mathrm{MZSP} 98238,1$ sh (F.S. Silva col. 1986); Caetité, $14^{\circ} 03^{\prime} 53.52^{\prime \prime} \mathrm{S}, 42^{\circ} 29^{\prime} 09.19^{\prime \prime} \mathrm{W}$, MZSP 29162, 15 sh (J. Vaz col. iii/1968); Mortugaba, $15^{\circ} 01^{\prime} 42.20^{\prime \prime} \mathrm{S}, 42^{\circ} 22^{\prime} 30.79^{\prime \prime} \mathrm{W}, \mathrm{MZSP} 143688,1 \mathrm{spm}$ (F.S.

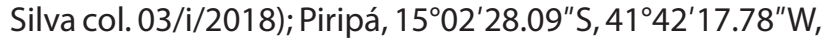

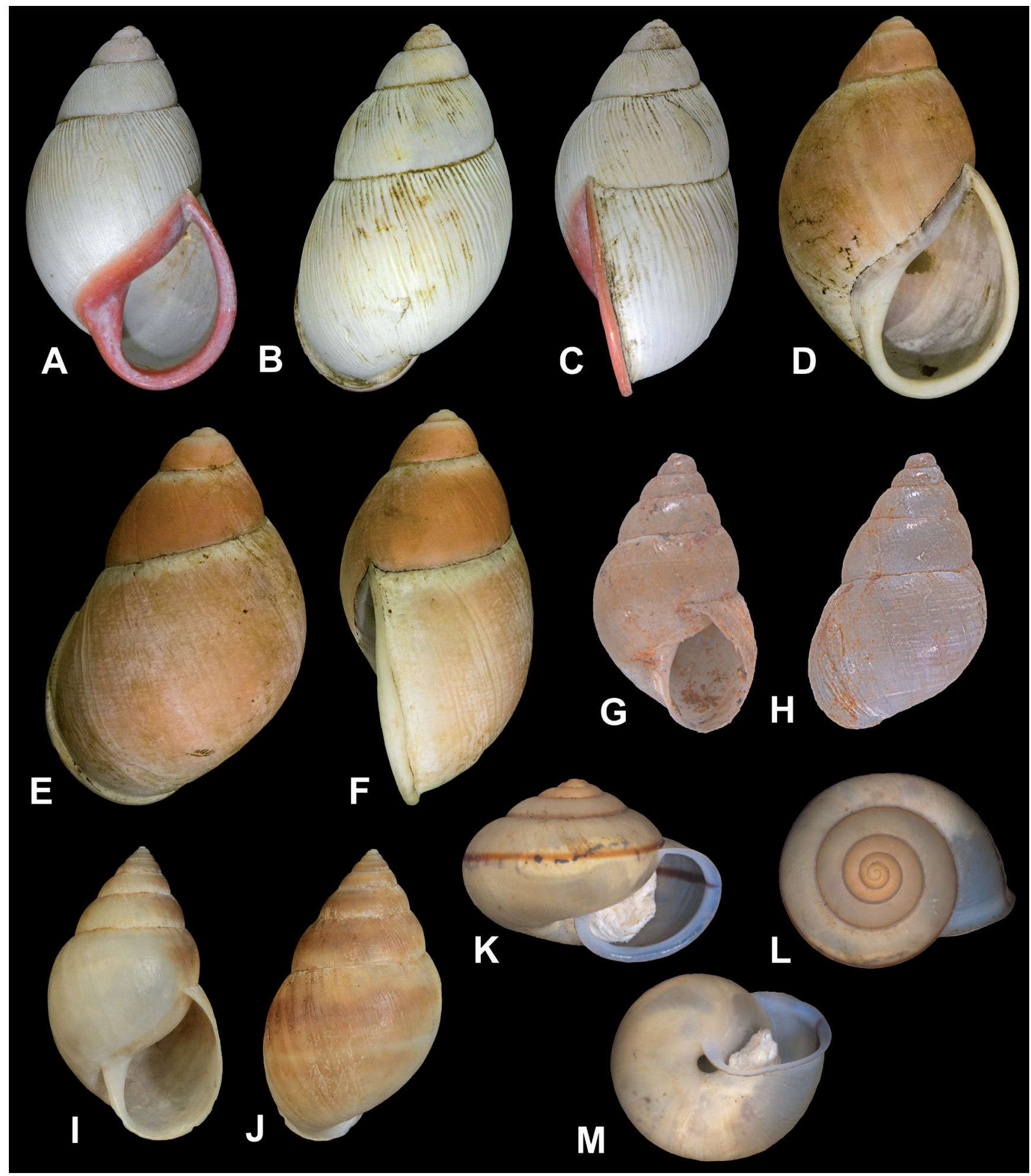

Figure 5. Shells from Bahia state. (A-C) Megalobulimus conicus, MZSP 136647, Cordeiros (H=90.9 mm). (D-F) Megalobulimus terrestris, MZSP 136671, Cordeiros ( $\mathrm{H}=95.5 \mathrm{~mm})$. (G-H) Bulimulus tenuissimus, MZSP 136599, Mortugaba ( $\mathrm{H}=8.4 \mathrm{~mm})$. (I-J) Rhinus durus, MZSP 144963, Cordeiros $(\mathrm{H}=32.7 \mathrm{~mm})$. (K-M) Bradybaena similaris, MZSP 136583, Piripá ( $D=11.6 \mathrm{~mm})$. 
MZSP 139661, 2 sh (F.S. Silva col. 07/viii/2018); Cordeiros, $15^{\circ} 02^{\prime} 23.90^{\prime \prime} \mathrm{S}, 41^{\circ} 56^{\prime} 02.07^{\prime \prime} \mathrm{W}, \mathrm{MZSP} 136647$, 4 sh (F.S. Silva col.17/i/2018);MZSP 136648, 2 sh (F.S. Silva col.03/i/2018); MZSP 136649, 1 sh (Doação, 2016); MZSP 136676, 1 spm (F.S. Silva col. 03/i/2018); MZSP 139662, 5 sh (F.S. Silva col. 04/viii/2018); MZSP 144961, 3 sh (ii/2019); Itapetinga, $15^{\circ} 14^{\prime} 51.08^{\prime \prime}$ S, $40^{\circ} 15^{\prime} 03.53^{\prime \prime}$ W, MZSP 102448, 1 sh (J.E. Santos col.).

Barcoding: GenBank reg nr MN688645 (MZSP 136674), MN688646 (MZSP 136676), MN756629 (MZSP 143688).

Remarks: This speces differs from Megalobulimus oblongus by its size $(70-100 \mathrm{~mm})$ and the last whorl of the teleoconch, which has pronounced axial riblets near the suture and more discreet riblets on the abapical portion of the whorl. Megalobulimus oblongus, on the other hand, has irregular malleated sculpture on its last whorls. Furthermore, it also displays a more defined umbilicus.

\section{Megalobulimus terrestris (Spix, 1827) Figs. 5D-F}

Synonymy, see Bequaert (1948: 108). Complement:

Strophocheilus (Megalobulimus) terrestris: Bequaert, 1948: 108, pl. 7, fig. 5, pl. 18, fig. 1; Martins, 1950: 143.

Strophocheilus cantagallanus: Morretes, 1949: 143; Jaeckel, 1952: 7.

Megalobulimus (Megalobulimus) cantagallanus: Morretes, 1953: 67; Oliveira \& Almeida, 1999: 29.

Megalobulimus (Megalobulimus) terrestris: Morretes, 1952: 113, 1953: 68; Oliveira \& Almeida, 1999: 30.

Megalobulimus terrestris: Oliveira et al., 1981:337; Salgado \& Coelho, 2003: 158; Simone, 2006: 220, fig. 834; Agudo-Padrón, 2014: 15; Breure \& Araújo, 2017: 107, fig. 39B; Lima et al., 2017: 329, fig. 2.

Bulimus terrestris: Fechter, 1983: 221.

Type locality: Bahia state, Brazil.

Occurrence: Brazil (Ceará, Rio Grande do Norte, Paraíba, Bahia, Rio de Janeiro, São Paulo, and Santa Catarina states) (Simone, 2006).

Materialexamined:BRAZIL:Bahia; Seabra, $12^{\circ} 28^{\prime} 00.00^{\prime \prime} \mathrm{S}$,

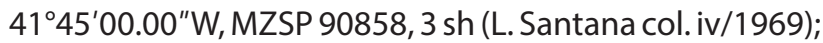
MZSP 29333, 8 sh (R. Santana col. iv/1965); Planaltino, $13^{\circ} 10^{\prime} 26.02^{\prime \prime} \mathrm{S}, 40^{\circ} 16^{\prime} 04.83^{\prime \prime} \mathrm{W}, \mathrm{MZSP}$ 18427, 5 sh (A. Silva. col. 1967); Ubaíra, $13^{\circ} 16^{\prime} 10.48^{\prime \prime}$ S, 39³9'44.79"W, MZSP 46480, 1 sh (J.E.A. Ranali col.); Jequié, $13^{\circ} 51^{\prime} 32.61^{\prime \prime S}$, 4005'01.59"W, MZSP 118328, 1 sh (M.U. Rodrigues col. 16/xii/1998); Caetité, 1403'53.52"S, 42²9'09.19"W, MZSP 90855, 1 sh (ex J.F. Vaz colln. xi/1969); Cordeiros, $15^{\circ} 02^{\prime} 23.90^{\prime \prime} \mathrm{S}, 41^{\circ} 56^{\prime} 02.07^{\prime \prime} \mathrm{W}, \mathrm{MZSP} 136671$, 1 sh (F.S. Silva col. 31/xii/2017).

Remarks: In contrast to the other two Megalobulimus species listed above, adults of $M$. terrestris have an olivaceous-brown periostracum with a few darker chest- nut-brown vertical streaks. Furthermore, the outer lip is white and the shell apex is short. Unfortunately, no fresh specimen was found for barcoding.

\section{Superfamily Orthalicoidea \\ Family Bulimulidae \\ Genus Bulimulus Leach, 1814 \\ Bulimulus tenuissimus (d'Orbigny, 1835)}

Figs. 5G-H

Helix tenuissima d'Orbigny, 1835b: 11; Moricand. 1836; 435; Breure, 1973: 123, 1979: 64.

Bulimus tenuissimus: Pfeiffer, 1848: 218; Reeve, 1849b: pl. 45, fig. 288; Albers, 1850: 167; Pfeiffer, 1853: 439, 1855: 160; Gray, 1854: 15; Hupé, 1857: 53; Pfeiffer, 1868: 152; Hidalgo, 1869: 119, 1870; 60; Duarte, 1971: 84.

Bulimus (Bulimulus) tenuissimus: Baker, 1913: 635; Beck, 1837: 67; Pfeiffer, 1842: 122.

Bulimus (Bulimulus) thoreyi Beck, 1837: 67; Pfeiffer, 1859: 500.

Bulimus corneus Potiez \& Michaud, 1838: 137, pl. 14, figs. 11-12.

Bulimus puellaris Reeve, 1849b: pl. 76, fig. 637; Pfeiffer, 1855: 160, 1868: 114.

Bulimulus tenuissimus: Smith, 1895: 316; Baker, 1926: 38, pl. 18, fig. 90; Jaekel, 1927: 137; Pilsbry, 1933: 69; Morretes, 1943: 116, 1949: 146; Barros-Araújo et al., 1960: 34; Oliveira et al., 1972: 16; Breure, 1976: 109; Tillier, 1980: 81; Oliveira et al., 1981: 345; Dutra, 1988: 583; Dutra-Clarke \& Souza, 1991: 292, fig. 1; Oliveira \& Oliveira, 1984: 11; Richardson, 1995: 63; Oliveira \& Almeida, 1999: 35; Salgado \& Coelho, 2003: 160; Silva \& Castro, 2003: 67; Simone, 2006: 120, fig. 370; Agudo-Padrón, 2008: 159, 2014: 15; Santos et al., 2010: 513; Agudo-Padrón \& Lenhard, 2011: 167; Salvador \& Simone, 2015: 3, fig. 6; Birckolz et al., 2016: 149, table 1; Salvador et al., 2018: 109, fig. 7 A.

Bulimulus (Bulimulus) tenuissimus: Pilsbry, 1897-1898: 64, pl. 10, figs. 91-92; Baker, 1913: 635; Haas, 1935, 111; Jaeckel, 1952: 7; Figueiras, 1963: 89.

Type locality: Rio de Janeiro state, Brazil.

Occurrence: Suriname, Bolivia, Brazil (Bahia, Espírito Santo, Maranhão, Pará, Pernambuco, Rio de Janeiro, São Paulo, Rio Grande do Sul, and Santa Catarina states), and Uruguay (Simone, 2006; Birckolz et al., 2016).

Material examined: BRAZIL: Bahia; Mortugaba, $15^{\circ} 01^{\prime} 42.20^{\prime \prime} \mathrm{S}, 42^{\circ} 22^{\prime} 30.79^{\prime \prime} \mathrm{W}, \mathrm{MZSP} 136597,1$ sh (F.S. Silva col. 04/i/2018); MZSP 136599, 2 sh (F.S. Silva col. 03/i/2018).

Remarks: The species is adaptated to living in urban areas and has already been introduced to North America (Salvador \& Simone, 2015). The original natural distribution is unknown, but it likely included Bahia state (Salvador \& Simone, 2015). 


\section{Family Simpulopsidae \\ Genus Rhinus Martens in Albers, 1960 \\ Rhinus durus (Spix, 1827)}

Figs. 5I-J

Bulimus durus Spix, 1827: 5, pl. 6, fig. 2; Pfeiffer, 1848: 104; Reeve, 1849b: pl. 40, fig. 253; Hupé, 1857: 43; Pilsbry, 1897-1898: 87, pl. 13, figs. 5-6, 24.

Rhinus durus: Albers, 1860: 223; Salgado \& Coelho, 2003: 163; Simone, 2006: 127, fig. 404.

Bulimulus durus: Bland \& Binney, 1873: 248; Oliveira et al., 2011: 87.

Bulimulus (Protoglyptus) durus: Haas, 1939: 269; Parodíz, 1944: 3; Jaeckel, 1952: 7.

Protoglyptus durus: Morretes, 1953: 70; Dutra-Clarke \& Souza, 1991: 294, pl. 2, fig. 5.

Protoglyptus (Protoglyptus) durus: Figueiras, 1962: 48.

Naesiotus durus: Breure, 1979: 69.

Type locality: Bahia state, Brazil.

Occurrence: Brazil (Ceará, Paraíba, Pernambuco, and Bahia states) (Simone, 2006).

Material examined: BRAZIL: Bahia; Brumado,

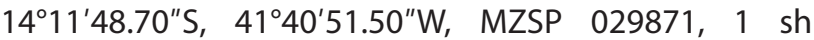
(L.S. Rocha col. 16/xii/1998); Cordeiros, $15^{\circ} 02^{\prime} 23.90^{\prime \prime} S$, $41^{\circ} 56^{\prime} 02.07^{\prime \prime}$, MZSP 144963, 1 sh (ii/2019).

\section{Superfamily Helicoidea Family Camaenidae \\ Genus Bradybaena Beck, 1837 \\ Bradybaena similaris (Férussac, 1821) \\ Figs. 5K-M}

Synonymy, see Silva et al. (2019b: 11). Complement:

Helix similaris: Férussac \& Deshayes, 1820-1851: 171 pls. 25, 27.

Bradybaena similaris: Burch, 1960: 26; Oliveira et al., 1981: 366; Scarabino, 2003: 206; Simone, 2006: 312, fig. 29; Thomé et al., 2006: 83; Santos et al., 2010: 516; Agudo-Padrón, 2011: 56, fig. 21, 2014: 18; Madella \& Auricchio, 2014: 55; Silva et al., 2019b: 11, fig. 4G-I.

Type locality: Timor.

Occurrence: Natural from Asia. Introduced in the USA (including Hawaii), Antilles, Venezuela, Brazil, Paraguay, and some Pacific Islands (Burch, 1960; Quintana, 1982; Cowie, 2001; Simone, 2006).

Material examined: BRAZIL: Bahia; Piripá, $15^{\circ} 02^{\prime} 28.09^{\prime \prime}$, 4142'17.78"W, MZSP 136583, 25 spm (F.S. Silva col. 08/i/2018).

Remarks: Live specimens were collected in urban areas. A fairly known worldwide invasive species, present inseveral states in eastern and southern Brazil, from Minas Gerais to Rio Grande do Sul (Morretes, 1949; Oliveira et al., 1981;
Agudo-Padrón \& Lenhard, 2011). The present record from Bahia represents a new occurrence and a northwards extension in the species distribution within Brazil.

\section{DISCUSSION}

In total 19 freshwater and land snail species were sampled in Condeúba, Cordeiros, Mortugaba and Piripá municipalites (Table 1). This number was complemented by two additional species from the historical collection of the MZSP (Asolene meta and Stenogyra octogyra), totaling 21 species and 10 families. Expeditions during the rainy season (December and January) evidenced a greater wealth of species, with a total of 17 species (Table 1). Even so, the rainy season was unusually dry during our colletion efforts (below the average values reported by Oliveira et al., 2017); the resulting dry soils and microhabitats could have influenced the number of species and of live specimens of land snails found (Pearce \& Örstan, 2006).

Caenogastropods showed little overall diversity in the material resulting from our collections, with three species, but Pomacea canaliculata was the most abundant species (Table 1). The freshwater pulmonates Biomphalaria glabrata and $B$. straminea were likewise

Table 1. Species collected in December/2017 to January/2018 (rainy season) and August/2018 (dry season). An asterisk (*) indicates a non-indigenous species.

\begin{tabular}{|c|c|c|c|}
\hline Taxon & Rainy season & Dry season & Total \\
\hline \multicolumn{4}{|l|}{ Ampullariidae } \\
\hline Pomacea canaliculata & 88 & 10 & 98 \\
\hline \multicolumn{4}{|l|}{ Hemisinidae } \\
\hline Aylacostoma sp. & 1 & 0 & 1 \\
\hline \multicolumn{4}{|l|}{ Thiaridae } \\
\hline *Melanoides tuberculata & 387 & 0 & 387 \\
\hline \multicolumn{4}{|l|}{ Physidae } \\
\hline Stenophysa marmorata & 2 & 9 & 11 \\
\hline \multicolumn{4}{|l|}{ Planorbidae } \\
\hline Biomphalaria glabrata & 2 & 8 & 10 \\
\hline Biomphalaria straminea & 37 & 4 & 41 \\
\hline \multicolumn{4}{|l|}{ Veronicellidae } \\
\hline Sarasinula plebeia & 5 & 0 & 5 \\
\hline \multicolumn{4}{|l|}{ Achatinidae } \\
\hline Beckianum beckianum & 0 & 11 & 11 \\
\hline Lamellaxis gracilis & 13 & 0 & 13 \\
\hline Leptinaria unilamellata & 1 & 0 & 1 \\
\hline * Lissachatina fulica & 1 & 0 & 1 \\
\hline *Opeas opella & 16 & 0 & 16 \\
\hline *Subulina octona & 2 & 0 & 2 \\
\hline \multicolumn{4}{|l|}{ Strophocheilidae } \\
\hline Megalobulimus conicus & 7 & 7 & 14 \\
\hline Megalobulimus oblongus & 3 & 1 & 4 \\
\hline Megalobulimus terrestris & 1 & 0 & 1 \\
\hline \multicolumn{4}{|l|}{ Bulimulidae } \\
\hline Bulimulus tenuissimus & 3 & 0 & 3 \\
\hline Rhinus durus & 1 & 0 & 1 \\
\hline \multicolumn{4}{|l|}{ Camaenidae } \\
\hline *Bradybaena similaris & 25 & 0 & 25 \\
\hline Total & 595 & 50 & 645 \\
\hline
\end{tabular}


abundant (Table 1). Among the Stylommatophora, the Achatinidae were the most diverse group, with a total of six species, albeit including introduced ones. The superfamily Orthalicoidea, typically diverse in the region, was collected in very small numbers, with only two species, one in each of the families Bulimulidae and Simpulopsidae. This is at odds compared to recent surveys and checklists in the country (e.g., Salvador et al., 2018; Lima et al., 2021), but might be an artifact of the unfavorable weather during the collection effort, as mentioned above. Microgastropods such as Punctoidea, Euconulidae and Scolodontidae, also abundant in others localites (e.g., Salvador et al., 2018), were not found in the present collections, despite leaf litter sampling. Once again, the dry soil might be a cause, as litter and soil snails tend to burrow deeper in the soil during dry periods (pers. obs.).

The genus Megalobulimus was surprisingly abundant and diverse in the present collections, with 29 especimens, especially by the large size and K-selection strategy of reproduction (Miranda \& Fontenelle, 2015). Megalobulimus conicus, in particular, was found in three of the four municipalities investigated.

Of all species recorded herein, five are non-indigenous. Of these, the records of Bradybaena similaris and Opeas opella represent important increments in these species' known distribution in Brazil, as these are the first records for Bahia state.

\section{ACKNOWLEDGMENTS}

This work was made possible with the assistance of residents of the municipalities visited, especially the Oliveira Santos family and Sérgio Ribeiro, for their dedicated efforts to support this study. The new collections were conducted under the license SiSBio 61707-1. F.S.S. received a graduate grant from the Coordenação de Aperfeiçoamento de Pessoal de Nível Superior, CAPES, Brazil (CAPES PROAP \#817757/2015).

\section{AUTHORS' CONTRIBUTIONS}

F.S.S.: Conceptualization, data curation, investigation, formal analysis, writing (original draft). L.R.L.S. Supervision, data curation, formal analysis, writing (review \& editing). R.B.S. Formal analysis, data curation, methodology, investigation, writing (review \& editing).

\section{REFERENCES}

Adams, H. 1866. List of land and freshwater shells collected by Mr. E. Barlett on the upper Amazonas and on the river Ucayali, eastern Peru with descriptions two species. Proceedings of the Zoological Society of London, (1866): 440-445+ pl. 38.

Aguayo, C.G. 1938. Los moluscos fluviatiles cubanos. Parte II - Sistemática. Memorias de la Sociedad Cubana de Historia Natural Felipe Poey, 12(3): 203-242.
Agudo-Padrón, A.I. 2008. Listagem sistemática dos moluscos continentais ocorrentes no estado de Santa Catarina, Brazil. Comunicaciones de la Sociedad Malacológica del Uruguay, 9(91): 147-179.

Agudo-Padrón, A.I. 2011. Threatened freshwater and terrestrial molluscs (Mollusca, Gastropoda et Bivalvia) of Santa Catarina State, Southern Brazil: check list and evaluation of regional threats. Biodiversity Journal, 2(2): 59-66.

Agudo-Padrón, A.I. 2014. Inventario sistemático de los moluscos continentales ocurrentes em el Estado de Santa Catarina, Brazil/Inventário sistemático dos moluscos continentais ocorrentes no Estado de Santa Catarina, Brazil. BIOMA, 2(21): 6-23.

Agudo-Padrón, A.I. \& Lenhard, P. 2011. Continental mollusc fauna of the Great Porto Alegre central region, RS, Southern Brazil. Biodiversity Journal, 2(4): 163-170.

Albers, J.C. 1850. Die Heliceen nach natürlicher Verwandtschaft: systematisch georduct. Berlin, Enslin. 262p.

Albers, J.C. 1860. Die Heliceen. Leipzig, Verlag von Wilhelm Engelmann. 359p.

Albuquerque, F.S.; Peso-Aguiar, M.C. \& Assunção-Albuquerque, M.J.T. 2008. Distribution, feeding behavior and control strategies of the exotic land snail Achatina fulica (Gastropoda: Pulmonata) in the northeast of Brazil. Brazilian Journal of Biology, 68(4): 837-842.

Albuquerque, F.S.; Peso-Aguiar, M.C.; Assunção-Albuquerque, M.J.T. \& Gálvez, L. 2009. Do climate variables and human density affect Achatina fulica (Bowditch) (Gastropoda: Pulmonata) shell length, total weight and condition factor? Brazilian Journal of Biology, 69(3): 879-885.

Almeida, M.N. \& Mota, G.G. 2011. Ecologia, reprodução e crescimento da concha de Beckianum beckianum Pfeiffer, 1846 (Pulmonata, Subulinidae) em condições naturais. Revista de Biologia e Farmácia, 6(1): 75-85.

Altena, C.O.v.R. 1960. On a small collection of land Mollusca from Surinam (Dutch Guyana). Basteria, 24(4): 48-51.

Andrews, K.L. \& Dundee, D. 1987. Las babosas veronicellidos de Centroamérica con énfasis en Sarasinula plebeia (= Vaginulus plebeius). (eiba, 28: 163-172.

Araújo, J.L.B. \& Bessa, E.C. 1993. Moluscos de importância econômica no Brazil. ii. Subulinidae, Subulina octona (Bruguière) (Mollusca, Gastropoda, Pulmonata, Stylommatophora). Revista Brazileira de Zoologia, 10(3): 489-497.

Arias, S. 1952. Algunos moluscos de la region de Baruta - el Hatillo. Memoria de la Sociedad de Ciências Naturales la Salle, 12(31): 47-65.

Arias, S. 1953. Algunos moluscos de la region de Perija. Memoria de la Sociedad de ciências Naturales la Salle, Caracas, 15(35): 245-257.

Baker, F. 1913. The land and freshwater mollusks of Stanford Expedition to Brazil. Proceedings Academy of Natural Sciences of Philadelphia, 65: 618-672, pls. 21-27.

Baker, H.B. 1926. The Mollusca Collected by the University of Michigan Williamson Expedition in Venezuela. Part IV. Occasional papers of the Museum of Zoology, University of Michigan, 167: 1-49.

Baker, H.B. 1927. The Mollusca collected by the University of Michigan Williamson Expedition in Venezuela. Part V. Occasional Papers of the Museum of Zoology, University of Michigan, 8(182): 1-36.

Baker, H.B. 1930. The Mollusca collected by the University of Michigan Williamson Expedition in Venezuela. Part VI. Occasional Papers of the Museum of Zoology, University of Michigan, 9(210): 1-95.

Baker, F.C. 1945a. The molluscan family Planorbidae. Urbana, University of Illinois Press. 530p.

Baker, H.B. 1945b. Some American Achatinidae. Nautilus, 58(3): 84-92.

Baker, H.B. 1961. Beckianum - new genus. Nautilus, 75(2): 84.

Barattini, L.P \& Ledón, D.A. 1949. Una nueva subespecie del genero Strophocheilus para la malaco-fauna uruguaya. Revista de la Sociedad Malacologica "Carlos de la Torre", 7(1): 1-2. 
Barbosa, C.S. 1992. Métodos de diagnósticos malacológico. Memórias do Instituto Oswaldo Cruz, 84 (suppl. 4): 311-313.

Barbosa, F.S. \& Dobbin-Jr., J.E. 1951. Estrutura interna dos Australorbis (Mollusca, Planorbidae) de Pernambuco e outros estados. Publicações Avulsas do Instituto Aggeu Magalhães, 1(1): 1-8.

Barbosa, F.S. \& Figueiredo, T. 1969. Geographical distribution of the snail hosts of Schistosomiasis mansoni in northeastern Brazil. Revista do Instituto de Medicina Tropical de São Paulo, 11(4): 285-289.

Barros-Araújo, J.L.; Rezende, H.E.B. \& Rodriguez, P.A.F. 1960. Sôbre Bulimulus tenuissimus (Orbgny, 1835) (Gastropoda, Pulmonata). Revista Brazileira de Biologia, 20(1): 33-42.

Basch, P.F. 1968. A scalariform Biomphalaria glabrata. Nautilus, 82(1): 21.

Beck, H.H. 1837. Index molluscorum praesentis aevis Musei Principis augustissimi Christiani Frederici. Hafniae. Fasciculus primus, 100p.

Bequaert, J.C. 1948. Monograph of the Strophocheilidae, a neotropical family of terrestrial mollusks. Bulletin of the Museum of Comparative Zoology, 100(1): $1-210+$ pls. 32.

Bequaert, J.C. 1950. Studies in the Achatininae, a group of African land snails. Bulletin of the Museum of Comparative Zoology at Harvard College, 105: $1-216$.

Bertoni, A.W. 1925. Sobre moluscos del Paraguay. Revista de la Sociedad Cientifica del Paraguay, 2(1): 71-73.

Bessa, E.C.A \& Araújo, J.L.B. 1995. Ocorrência de autofecundação em Subulina octona (Brugüière) (Pulmonata, Subulinidae) em condições de laboratório. Revista Brazileira de Zoologia, 12(3): 719-723.

Bigarella, J.J. 1951. Contribuição ao estudo dos sambaquís no Estado do Paraná. 1 - Regiões adjacentes às baías de Paranaguá e Antonina. Arquivos de Biologia e Tecnologia de Curitiba, 5-6: 231-292 + pls. 50-57.

Birckolz, C.J.; Salvador, R.B.; Cavallari, D.C. \& Simone, L.R.L. 2016. Illustrated checklist of newly described (2006-2016) land and freshwater Gastropoda from Brazil. Archiv für Molluskenkunde, 145(2): 133-150.

Bland, T. \& Binney, W.G. 1873. On the lingual dentition and jaw of certain terrestrial pulmonata from the United States, with remarks on their systematic value. Proceedings of the Academy of Natural Sciences of Philadelphia, 25(2): 240-256.

Boffi, A.V. 1979. Moluscos brasileiros de interesse médico e econômico. São Paulo, Ed. Hucitec, FAPESP. 182p.

Bouchet, P.; Rocroi, J.P.; Hausdorf, B.; Kaim, A.; Kano, Y.; Nützel, A.; Parkhaev, P.; Schrödl, M. \& Strong, E.E. 2017. Revised classification, nomenclator and typification of gastropod and Monoplacophoran families. Malacologia, 61(1-2): 1-526.

Bowdich, T.E. 1822. Elements of conchology, including the fossil genera and the animals, 1. Univalves. Paris, Smith. 80p +19 pls.

Brandt, R.A.M. 1974. The non-marine aquatic Mollusca of Thailand. Archiv für Molluskenkunde, 105(1-4): 1-423.

Breure, A.S.H. 1973. Index to the neotropical land Mollusca describes by Alcide d'Orbigny, with notes on localities of the mainland species. Basteria, 37(5-6): 113-135.

Breure, A.S.H. 1976. Notes on Bulimulidae (Gastropoda, Euthyneura), 4. Some Bulimulidea from French Guyana and Surinam, with notes on their anatomy. Zoologische Mededelingen, 50(7): 107-115 + 1 pl.

Breure, A.S.H. 1979. Systematics, phylogeny and zoogeography of Bulimulinae (Mollusca). Zoologische Verhandelingen, 168: 1-215.

Breure, A.S.H. \& Araújo, R. 2017. The Neotropical land snails (Mollusca, Gastropoda) collected by the 'Comisión Científica del Pacífico.' PeerJ, 5: $1-142$.

Breure, A.S.H.; Abletti, J.D. \& Audibert, C. 2020. A proposed solution to a lengthy dispute: what is Leptinaria (uni)lamellata (Mollusca, Gastropoda, Achatinidae)? Folia Conchyliologica, 58: 23-31.
Breure, A.S.H.; Fields, A.; Hovestadt, A. \& Robinson, D.G. 2016. The land Mollusca (Gastropoda) of Saint Kitts and Nevis (Lesser Antilles), with description of a new species. Nautilus, 130(2): 27-52.

Bruguière, J.G. 1792. Encyclopédie méthodique ou par ordre de matières. Histoire naturelle des vers. Paris, Pancoucke. v. 1,757p.

Burch, J.B. 1960. Some snail $s$ and slugs of quarantine significance to the United States. Sterkiana, 2: 13-53.

Capinera, J.L. 2017. Biology and food habits of the invasive snail Allopeas gracile (Gastropoda: Subulinidae). Florida Entomologist, 100(1): 116-123.

Carvalho, C.M.; Silva, J.P.; Mendonça, C.L.F.; Bessa, E.C.A. \& D'Ávila, S. 2009. Life history strategy of Leptinaria unilamellata (d'Orbigny, 1835) (Mollusca, Pulmonata, Subulinidae). Invertebrate Reproduction and Development, 53(4): 211-222.

Carvalho, 0.D.S.; Mendonça, C.L.F.; Marcelino, J.M.D.R.; Passos, L.K.J.; Fernandez, M.A.; Leal, R.S.; Caldeira, R.L.; Scholte, R.G.C.; Carmo, E.H.; Mesquita, S.G. \& Thiengo, S.C. 2018. Geographical distribution of intermediate hosts of Schistosoma mansoni in the states of Paraná, Minas Gerais, Bahia, Pernambuco and Rio Grande do Norte, Brazil, 2012-2014. Epidemiologia e Serviços de Saúde, 27(3): 1-9.

Carvalho, 0.S. 1986. Ocorrência de um tiarídeo (Mollusca) no lago da Pampulha Belo Horizonte, MG, Brazil. Revista da Sociedade Brasileira de Medicina Tropical, 19: 57.

Carvalho, 0.S.; Nunes, I.M. \& Caldeira, R.L. 1998. First Report of Biomphalaria glabrata in the State of Rio Grande do Sul, Brazil. Memórias do Instituto Oswaldo Cruz, 93(1): 39-40.

Castellanos, Z.J.A. \& Fernández, D. 1976. Moluscos Gasteropodos. Ampullariidae. In: Ringuelet, R.A. Fauna de agua dulce de la Republica Argentina. Buenos Aires, FECIC. v. 15, 33p.

Cavallari, D.C.; Salvador, R.B. \& Simone, L.R.L. 2016. A possible land snail diversity hotspot in Bahia state, Brazil. Tentacle, 24: 14-16.

Clench, W.J. 1936. The Physidae of the West Indies. Memorias de la Sociedad Cubana de Historia Natural, 10: 335-342 + 15 pl.

Clessin, S. 1888. Binnenmollusken aus SüdBrazilien. Malakozoologische Blätter, 10(2): 165-174.

Coimbra-Jr.; C.E.A. \& Santos, R.V. 1986. Moluscos aquáticos do Estado de Rondônia (Brazil), com especial referência ao Genus Biomphalaria Preston, 1910 (Pulmonata, Planorbidae). Revista de Saúde Pública, 20(3): 227-234.

Coomans, H.E. 1967. The non-marine Mollusca of St. Martin (Lesser Antilles). Studies on the Fauna of Curacao and other Caribbean Islands, 24: 118-145. (Uitgaven Natuurwetenschappelijke Studiekring voor Suriname en de Nederlandse Antillen 46)

Cowie, R.H. 1998. Patterns of introduction of non-indigenous non marine snails and slugs in the Hawaiian Islands. Biodiversity and Conservation, 7(3): 349-368.

Cowie, R.H. 2001. Invertebrate invasions on Pacific Islands and the replacement of unique native faunas: a synthesis of the land and freshwater snails. Biological Invasions, 3(2): 119-136.

Cowie, R.H. \& Thiengo, S.C. 2003. The apple snails of the Americas (Mollusca: Gastropoda: Ampullariidae: Asolene, Felipponea, Marisa, Pomacea, Pomella): a nomenclatural and type catalog. Malacologia, 45: 41-100.

Cowie, R.H.; Kabat, A.R \& Evenhuis, N.L. 2001. Ampullaria canaliculata Lamarck, 1822 (currently Pomacea canaliculata; Mollusca, Gastropoda): proposed conservation of the specific name. Bulletin of Zoological Nomenclature, 58: 13-18.

Cuezzo, M.G. \& Drahg, F. 1995. Moluscos depositados en la Colección de la Fundación Miguel Lillo: Argentina. Acta Zoológica Lilloana, 43(1): 185-205.

D’Ávila, S.; Medeiros, C.; Vargas, T. \& Mendonça, C.L.F. 2018. Life history of Subulina octona (Brugüière) (Gastropoda: Pulmonata: Subulinidae) 
based on four-year laboratory observations and a comparative histological analysis of egg-retaining and ovoviviparous subulinids. Journal of Natural History, 52(23-24): 1551-1559.

Dall, W.H. 1896. Insular land shells faunas, especially as illustrated by the data obtained by Dr. G. Baur in the Galapagos Island. Proceedings of the Academy of Natural Sciences of Philadelphia, 48: 395-460.

Darrigran, G. 1991. Análisis de la malacofauna de los arroyos afluentes del estuario del Río de la Plata. República Argentina. Biología Acuática, 15(2): 212-213.

Darrigran, G.; Agudo-Padrón, I.; Baez, P.; Belz, C.; Cardoso, F.; Carranza, A.; Collado, G.; Correoso, M.; Cuezzo, M.G.; Fabres, A.; Gregoric, D.E.G.; Letelier, S.; Ludwig, S.; Mansur, M.C.; Pastorino, G.; Penchaszadeh, P.; Peralta, C.; Rebolledo, A.; Rumi, A.; Santos, S.; Thiengo, S.; Vidigal, T. \& Damborenea, C. 2020. Non-native mollusks throughout South America: emergent patterns in an understudied continent. Bilogical Invasions, 1-19.

Deisler, J.E. \& Stange, L.A. 1982. The giant South American snail, Megalobulimus oblongus (Muller) (Gastropoda: Megalobulimidae). Entomology Circular, 239: 1-4.

Delannoye, R.; Charles, L., Pointier, J.P. \& Massemin, D. 2015. Mollusques continentaux de la Martinique. Paris, Muséum national d'Histoire Naturelle. 328p.

Díaz, A.C. \& Martín, S.M. 2013. Biodiversity of molluscs in the Multiple-Use Natural Reserve Guillermo Enrique Hudson in Florencio Varela, Buenos Aires, Argentina. Check List, 9(1): 25-27.

Duarte, E. 1971. Lo nuestro em "Los moluscos del viaje al Pacifico" de Hidalgo y Martinez. Comunicaciones de la Sociedad Malacológica del Uruguay, 20(3): 83-89.

Dunker, W. 1848. Diagnoses specierum novarum generis Planorbis collectionis Cumingianae. Proceedings of the Malacological Society of London, 16: 40-43.

Dutra, A.V.C. 1988. Aspectos da ecologia e da reprodução de Leptinaria uniamellata (Orbigny, 1835) (Gastropoda, Subullnidae). Revista Brasileira de Zoologia, 5(4): 581-591.

Dutra-Clarke, A.V.C. \& Souza, F.B.V.A. 1991. Bulimulidae (Gastropoda, Stylomatophora) do Nordeste do Brazil. Revista Brasileira de Zoologia, 7(3): 289-304.

Ernest, A. 1948. Enumeracion sistematica de las especies de moluscos terrestres y de agua dulce, halladas hasta ahora em los alrededores de Caracas y demas partes de la Republica. Revista de la Sociedad Malacologica "Carlos de la Torre", 6(2): 73-82.

Estebenet, A.L.; Martín P.R. \& Burela S. 2006. Conchological variation in Pomacea canaliculata and other South American Ampullariidae (Caenogastropoda, Architaenioglossa). Biocell, 30(2): 329-335.

Eston, M.R.; Menezes, G.V.; Antunes, A.Z.; Santos, A.S.R. \& Santos, A.M.R. 2006. Espécie invasora em unidade de conservação: Achatina fulica (Bowdich, 1822) no parque estadual Carlos Botelho, Sete Barras, SP, Brazil (nota científica). Revista do Instituto Florestal, 18: 173-179.

Fechter, R. 1983. Liste des Typenmaterials der von J.B. v. Spix in Brazilien gesammelten Unionacea (Mollusca/Bivalvia). Spixiana, 9(suppl): 221-223.

Fernandez, D. \& Castellanos, Z.J.A. 1973. Clave generica de la malacofauna terrestre argentina. Revista del Museo de la Plata, Nueva serie, 11(107): 265-285.

Fernandez, M.A.; Thiengo, S.C. \& Boaventura, M.F. 2001. Gastrópodes límnicos do Campus de Manguinhos, Fundação Oswaldo Cruz, Rio de Janeiro, RJ. Revista da Sociedade Brasileira de Medicina Tropical, 34(3): 279-282.

Fernandez, M.A.; Thiengo, S.C. \& Simone, L.R.L. 2003. Distribution of the introduced freshwater snail Melanoides tuberculatus (Gastropoda, Thiaridae) in Brazil. Nautilus, 117(3): 78-82.
Ferrari, A.A. \& Hofmann, P.R.P. 1992. First register of Biomphalaria straminea Dunker, 1848, in Santa Catarina State. Revista do Instituto de Medicina Tropical de São Paulo, 34(1): 33-35.

Férussac, A.E.J.P.J.F.A. 1821. Tableaux systématiques des animaux mollusques classés en familles naturalles, dans lesquels on a établi la concordance de tous les systèmes; suivis d'un prodrome général pour tous les mollusques terrestres ou fluviatiles, vivants ou fossilles. Deuxième partie (Première section) Tableaux particuliers des mollusques terrestres et fluviatiles..., Classe des Gasterópodes... II Tableaux de la famille des Limaçons. Paris, Arthus Bertrand. 94p.

Férussac, D. \& Deshayes, G.P. 1820-1851. Histoire naturelle générale et particuliére des mollusques terrestres et fluviatiles. Paris, Chez J.B. Baillière. v. 2, 868p. +171 pls.

Figueiras, A. 1962. Sobre la presencia del género Protoglyptus Pilsbry em el Uruguay (Bulimulinae). Comunicaciones de la Sociedad Malacologica del Uruguay, 1(2): 47-48.

Figueiras, A. 1963. Enumeracíon sistemática de los moluscos terrestres del Uruguay. Comunicaciones de la Sociedad Malacologica del Uruguay, 1(4): 79-96.

Figueiredo, C.C.S.B. 1989. Dispersão de Biomphalaria straminea no estado de Sergipe: um estudo comparativo com dezenove anos de intervalo. Memórias do Instituto Oswaldo Cruz, 84(3): 383-387.

Fiori, A.M.C. \& Jaeger, C.P. 1978. Biomass estimation of a stylomatophoran snail. Revista Brasileira de Biologia, 38(4): 847-849.

Fischer, M.L. \& Colley, E. 2004. Diagnóstico da ocorrência do caramujo gigante africano Achatina fulica Bowdich, 1822 na APA de Guaraqueçaba, Paraná, Brazil. Revista Estudos de Biologia, 26(54): 43-50.

Fischer, M.L. \& Colley, E. 2005. Espécie invasora em reservas naturais: caracterização da população de Achatina fulica Bowdich, 1822 (Mollusca - Achatinidae) na Ilha Rasa, Guaraqueçaba, Paraná. Brazil. Biota Neotropica, 5(1): 123-144.

Fischer, M.L.; Simião, M.; Colley, E.; Zenni, R.D.; Silva, D.A.T. \& Latoski, N. 2006. 0 caramujo exótico invasor na vegetação nativa em Morretes, PR: diagnóstico da população de Achatina fulica Bowdich, $1822 \mathrm{em}$ um fragmento de Floresta Ombrófila Densa aluvial. Biota Neotropica, 6(2): 1-5.

Folmer, 0.; Black, M.; Hoeh, W.; Lutz, R. \& Vrijenhoek, R. 1994. DNA primers for amplification of mitochondrial cytochrome $c$ oxidase subunit I from diverse metazoan invertebrates. Molecular Marine Biology and Biotechnology, 3: 294-299.

França, R.S.; Suriani, A.L. \& Rocha, 0. 2007. Composição das espécies de moluscos bentônicos nos reservatórios do baixo rio Tietê (São Paulo, Brazil) com uma avaliação do impacto causado pelas espécies exóticas invasoras. Revista Brasileira de Zoologia, 24(1): 41-51.

Freitas, J.R.; Bedê, L.C.; Marco-Jr., P.; Rocha, L.A. \& Santos, M.B.L. 1987. Population dynamics of aquatic snails in Pampulha reservoir. Memórias do Instituto Oswaldo Cruz, 82(4): 299-305.

Gnaspini, P. \& Trajano, E. 1994. Brazilian cave invertebrates with a checklist of troglomorphic taxa. Revista Brasileira de Entomologia, 38(4): 549-584.

Gomes, S.R.\&Thomé, J.W. 2004. Diversity and distribution of the Veronicellidae (Gastropoda, Soleolifera) in the Oriental and Australian biogeographical regions. Memoirs of the Queensland Museum, 49: 589-601.

Gomes, S.R.; Robinson, D.G.; Zimmerman, F.J.; Obregón, 0. \& Barr, N.B. 2013. Morphological and molecular analysis of the Andean slugs Colosius confusus, n. sp., a newly recognized pest of cultivated flowers and coffee from Colombia, Ecuador and Peru, and Colosius pulcher (Colosi, 1921) (Gastropoda, Veronicellidae). Malacologia, 56: 1-30.

Gómez, J.D.; Vargas, M. \& Malek, E.A. 1986. Freshwater mollusks of the Dominican Republic. Nautilus, 100(4): 130-134.

González-Valdivia, N.A.; Martínez-Puc, J.F. \& Echavarría-Góngora, E.J. 2018. Malacofauna en dos sistemas silvopastoriles en Estelí, Nicaragua. Journal of the Selva Andina Animal Science, 5(1): 3-13. 
Gordillo, S. 2018. El caracol gigante Megalobulimus lorentzianus: uma especie biocultural de la Provincia de Córdoba. Revista Facultad de Ciencias Exactas, Físicas y Naturales, 5(2): 63-69.

Götting, K.L. 1978. Lista preliminar de los caracoles terrestres de la region septentrional de Colombia. Anales del Instituto de Investigaciones Marinas de Punta de Betin, 10: 101-110.

Graeff-Teixeira, C. 2007. Expansion of Achatina fulica in Brazil and potential increased risk for angiostrongyliasis. Transactions of the Royal Society of Tropical Medicine and Hygiene, 101: 743-744.

Gray, L.E. 1854. List of the shells of South America in the collection of the British Museum collected and described by Alcide d' Orbigny in the "Voyage dans I'Amérique Méridionale". London, Taylor and Francis. 89p.

Gregoric, D.E.G.; Núez-Rumi, V. \& Roche, M.A. 2006. Freshwater gastropods from del Plata Basin, Argentina. Checklist and new locality records. Comunicaciones de la Sociedad Malacológica del Uruguay, 9(89): 51-60.

Guilding, L. 1828. Observations on the zoology of the Caribbean Islands. Zoological Journal, London, 3: 527-544.

Haas, F. 1935. Kurze bemerkungen V (3): binnenschnecken aus verschiedenen teilen Braziliens. Archiv für Molluskenkunde, 67(3): 109-111.

Haas, F. 1939. Zurkenntnis der binnen-Mollusken N.0.-Brazilien. Senckenbergiana, 21(3-4): 254-278.

Haas, F. 1949. Land-und Süßwassermollusken aus dem Amazonas-Gibiete. Archiv für Molluskenkund, 78(4-6): 149-156.

Haas, F. 1953. Mollusks from Ilha Grande, Rio de Janeiro, Brazil. Fieldiana (Zoology), 34(20): 203-209.

Haas, F. 1959. Inland mollusks from Venezuela, Southern Brazil and Peru. Fieldiana (Zoology), 39(31): 363-371.

Hayes, K.A.; Cowie, R.H.; Thiengo, S.C. \& Strong, E.E. 2012. Comparing apples with apples: clarifying the identities of two highly invasive Neotropical Ampullariidae (Caenogastropoda). Zoological Journal of the Linnean Society, 166: 723-753.

Hidalgo, J.G. 1869. Moluscos del viaje al Pacifico verificado de 1862 a 1865 por uma comision de naturalistas enviada por el gobierno Español. Parte primeira univalvos terrestres. Madrid, Miguel Ginesta. $152 \mathrm{p}+8 \mathrm{pls}$.

Hidalgo, J.G. 1870. Catalogue des coquilles terrestres recueillis par les naturalistes de la commission scientifique espagnole sur divers points de I'Amérique meridionale. Journal de Conchyliologie, 18: 27-70.

Hoffmann, H. 1925. Die Vaginuliden. Ein Beitrag zur Kenntnis ihre Biologie, Anatomie, Systematik, geographischen Verbreitung und Phylogenie. Jenaische Zeitschrift für Naturwissenschaft, 61(1/2): 1-374.

Hupé, H. 1857. Mollusques. In: Castelnau F de (Ed.). Animaux nouveaux ou rares recueillis pendant l'expédition dans les parties centrales de I'Amérique du Sud, de Rio de Janeiro à Lima, et de Lima au Para; par ordre gouvernement Français pendant les années 1843 à 1847, Partie 7, Tome 3, partie 3. Paris, Bertrand, 103p.

Instituto Brasileiro de Geografia e Estatística (IBGE). 1990. Divisão Regional do Brasil em Mesorregiões e Microrregiões Geográficas. Rio de Janeiro, IBGE. $135 p$.

Ihering, H.v. 1915. Molluscos. Publicação da Commissão de Linhas Telegraphicas Estrategicas de Matto-Grosso ao Amazonas, 5(22): 1-13.

Jaeckel, S. 1927. Die mollusken der müllegger'schen Brasilenexpedition. Zoologischer Anzeiger, 75(5-8): 129-139.

Jaeckel, S. 1952. Short review of the land - and freshwater molluscs of the Nort-East States of Brazil. Dusenia, 3(1): 1-10.

Jay, J.C. 1839. A catalogue of the shells, arranged according to the Lamarckian system. New York, Wiley \& Putnam. 82p.

Karlin, E.J. 1956. Notes on the ecology of Zonitoides arboreus (Say), Opeas pumilum (Pfeiffer), and Lamellaxis gracilis (Hutton) in greenhouses. The American Midland Naturalist, 55(1): 121-125.
Klappenbach, M.A. 1967. La primeira lista de moluscos publicada em el Uruguay. Comunicaciones de la Sociedad Malacologica del Uruguay, 2(12): 41-44.

Klappenbach, M.A. 1997. Origen y desarrollo de la Malacologia em el Uruguay. Comunicaciones de la Sociedad Malacologica del Uruguay, 8(72-73): 45-56.

Kloetzel, K. 1959. Resistência à dessecação do Australorbis glabratus. Comparação entre os caramujos de Olinda (Pernambuco) e um dos focos de Salvador (Bahia). Revista do Instituto de Medicina Tropical de São Paulo, 4(5): 296-298.

Kosloski, M.A. \& Fischer, M.L. 2002. Primeira ocorrência de Achatina fulica (Bowdich, 1822) no litoral do estado do Paraná (Mollusca; Stylomatophora; Achatinidae). Estudos de Biologia, 24(49): 65-69.

Kotzian, C.B. \& Amaral, A.M.B. 2013. Diversity and distribution of mollusks along the Contas River in a tropical semiarid region (Caatinga), Northeastern Brazil. Biota Neotropica, 13(4): 299-314.

Leme, J.L.M. 1966. Sobre A Ocorrência do Subgenero Physella no Brazil, e descrição de uma nova espécie (Mollusca, Gastropoda). Papeis Avulsos de Zoologia, 19(27): 269-278.

Leme, J.L.M. 1975. A fauna malacológica do material recolhido pela Missão Arqueológica Franco-Brasileira em Lagoa Santa, Minas Gerais. Arquivos Museu Nacional, Rio de Janeiro, 55: 174.

Lim, W.H.; Li, T.J. \& Cai, Y. 2018. Diversity of terrestrial snails and slugs in Nee Soon freshwater swamp forest, Singapore. Gardens' Bulletin Singapore, 70(suppl. 1): 109-121.

Lima, D.M.; Tenório, S.; Gomes, K.0;; Filadelfo, T. \& Salvador, R.B. 2017. Observation of scavenging events on shells of Megalobulimus (Gastropoda, Pulmonata) by Lear's macaws. The Festivus, 49(4): 328-331.

Lima, L.C. 1995a. Familys Chilinidae, Ancylidae, Physidae e Lymnaeidae. In: Barbosa, F.S. Tópicos de Malacologia Mádica. Rio de Janeiro, Editora FIOCRUZ. p. 80-89.

Lima, L.C. 1995b. Family Planorbidae. In: Barbosa, F.S. Tópicos de malacologia médica. Rio de Janeiro, Editora FIOCRUZ. p. 90-112.

Lima, MS.; Silva, FS.; Simone, LRL.; Salvador, RB. \& Guilherme, E. 2021. Terrestrial gastropods of Humaitá Forest Reserve, southwestern Brazilian Amazon. Check List, 17(1): 269-281.

Lopes, H.S. 1957. Relação dos moluscos coletados na Excursão às Zonas das Estradas de Ferro Noroeste do Brazil e Brazil-Bolívia, nos estados de São Paulo e de Mato Grosso, Brazil e Bolívia. Publicações Avulsas do Museu Nacional, 20: 43-44.

Lucena, D.T. 1951. Lista de moluscos do Nordeste, com um apêndice sobre algumas espécies de outras regiões. Papéis Avulsos do Departamento de Zoologia, 10(3): 93-104.

Lucena, D.T. 1953. Chave provisória para diagonose das espécies Brazileiras de moluscos planorbídeos. Revista Breasileira de Malariologia e Doenças Tropicais, 5(3): 245-248.

Lucena, D.T. 1956. Resenha sistemática dos planorbídeos Brazileiros. Publicações Avulsas do Centro de Pesquisas Aggeu Magalhães, 6: 1-104.

Lutz, A. 1918. On Brazilian fresh-water shells of the genus Planorbis. Memórias do Instituto Oswaldo Cruz, 10(1): 45-61.

Luz, E.; Silva, S.M.; Carvalho, A.P. \& Castro, N. 1998. Atualização da sistemática e de planorbídeos (Gasteropoda, Pulmonata) no Estado do Paraná (Brazil). Acta Biologica Paranaense, 27: 39-55.

Luz, E.; Stumpf, I.V.K. \& Borba, A.M. 1981. Desaparecimento da Biomphalaria glabrata (Say, 1818) de Curitiba, Paraná. Acta Biologica Paranaense, 10-11: 107-112.

Lydeard, C.; Cowie, R.H.; Bogan, A.E.; Bouchet, P.; Cummings, K.S.; Frest, T.J.; Herbert, D.G.; Hershler, R.; Gargominy, 0.; Perez, K.; Ponder, W.F.; Roth, B.; Seddon, M.; Strong, E.E. \& Thompson, F.G. 2004. The global decline of nonmarine mollusks. BioScience, 54: 321-330. 
Maceira, F.D. 2003. Las especies de la familia Veronicellidae (Mollusca, Soleolifera) en Cuba. Revista de Biologia Tropical, 51(3): 453-461.

Madella, C.R. \& Auricchio, P. 2014. Record of Achatina fulica Bowdich (Mollusca, Gastropoda) in the Cerrado-Caatinga ecotone in southern Piauí State, Brazil. Acta Biológica Paranaense, 43(1-2): 53-60.

Magalhães, L.A. 1964. Moluscos planorbídeos do estado da Guanabara. Revista Brasileira de Biologia, 24(3): 277-288.

Mansur, G.G. \& Thomé, J.W. 1994. Contribuição à biologia de Sarasinula linguaeformis (Semper, 1885) e Sarasinula plebeia (Fischer, 1868) (Veronicellidae, Gastropoda). Biociências, Porto Alegre, 2(2): 39-47.

Marcus, E. \& Marcus, E. 1968. Über einige Subulinidae (Pulmonata von São Paulo). Beitrage zur Neotropischen Fauna, 5: 186-208.

Martello, A.R.; Nunes, I.G.W.; Boelter, R.A. \& Leal, L.A. 2008. Malacofauna límnica associada à macrófitas aquáticas do rio Iguariaçá, São Borja, RS, Brazil. Ciência e Natura, 30(1): 27-41.

Martens, E.v. 1868. Ueber südBrazilianishe land- und süsswassermollusken. Malakozoologische Blätter, 15: 169-217.

Martens, E.v. 1873. Die binnenmollusken Venezuela's. In: Reichert, K.B. (Ed.). Fertschriftzur Feierdes hundertgähringen Bestehens. Gesellschaft Naturfoschender Freunde zu Berlin. Berlin. p. 157-225 + 2 pls.

Martins, A.V. 1938. Contribuição ao estudo do genêro Australorbis Pilsbry, 1934. Memórias do Instituto Biológico Ezequiel Dias, 2: 5-61.

Martins, E.A. 1950. Nota sôbre os moluscos coletados por Richard Rathbun, em 1876, na praia Consolidada e Sublevada e no sambaqui de Porto Santo, Ilha de Itaparica, Estado da Bahia. Revista Brasileira de Biologia, 10(1): 141-143.

Martins-Silva, M.J. \& Barros, M. 2001. Occurrence and distribution of freshwater molluscs in the Riacho Fundo Creek Basin, Brazilia, Brazil. Revista de Biología Tropical, 49(3-4): 865-870.

Massemin, D.; Lamy, D.; Pointier, J.P. \& Gargominy, 0. 2009. Coquillages et escargots de Guyane. Paris, Muséum National d'Histoire Naturelle. 456p.

Michelson, E.H. \& DuBois, L. 1974. Lymnaea emarginata, a possible agent for the control of the schistosome-snah host, Biomphalaria glabrata. Nautilus, 88(4): 101-108.

Miquel, S.E. \& Herrera, H.W. 2014. Catalogue of terrestrial gastropods from Galápagos (except Bulimulidae and Succineidae) with description of a new species of Ambrosiella Odhner (Achatinellidae). Archiv für Molluskenkunde, 143(2): 107-133.

Miquel, S.E. \& Jaime, G.L. 2018. Subulinidae snails from northern Argentina with description of a new species of Nannobeliscus Weyrauch. Spixiana, 41(1): 1-7.

Miranda, M.S. \& Fontenelle, J.H. 2015. Population dynamics of Megalobulimus paranaguensis in the Brazilian southeast coast. Zoologia, Curitiba, 32: 463-468.

Moricand, S. 1836. Mémorie sur les coquilles terrestres et fluviatiles, envoyées de Bahia par M.J. Blanchet. Memoires de la Société de Physique et d'Histoire Naturelle de Genève, 7(2): 415-446.

Morretes, F.L. 1943. Contribuição ao estudo da fauna Brasileira de moluscos: resultados de uma pequena coleção de moluscos obtida pela excursão científica realizada pelo Instituto Osvaldo Cruz em outubro de 1938. Papéis Avulsos do Departamento de Zoologia, 3(7): 111-126.

Morretes, F.L. 1949. Ensaio de Catálogo dos Moluscos do Brasil. Arquivo do Museu Paranaense, 7: 1-216.

Morretes, F.L. 1952. Novas espécies brasileiras da Family Strophocheilidae. Arquivos de Zoologia do Estado de São Paulo, 8(4): 109-126.

Morretes, F.L. 1953. Addenda e corrigenda ao ensaio de catálogo dos moluscos do Brazil. Arquivo do Museu Paranaense, 10: 37-76.

Müller, 0.F. 1774. Vermium terrestrium et fluviatilium seu animalium infusoriorum, helminthicorum et testaceorum, non marinorum, sucinta historia, Haviniae \& Lipsiae. 2v.
Naranjo-Garcia, E.; Thome, J.W. \& Castillejo, J. 2007. A review of the Veronicellidae from Mexico (Gastropoda: Soleolifera). Revista Mexicana de Biodiversidad, 78(1): 41-50.

Núñes, V. 2011. Revisión de dos especies de Physidae. Revista Mexicana de Biodiversidad, 82: 93-108.

Oliveira, M.P. \& Almeida, M.N. 1999. Conchas dos caramujos terrestres do Brazil. Juiz de Fora, Editora Associada. 61p.

Oliveira, M.P. \& Oliveira, M.H.R. 1984. Comunicações malacológicas № 16. Listagem de tipos de gastropoda pulmonata Brazileiros depositados em quatro museus europeus. Boletim do Instituto de Ciências Biológicas e de Geociências, 38: 1-46.

Oliveira, M.P.; Rezende, G.J.R. \& Castro, G.A. 1981. Catálogo dos moluscos da Universidade Federal de Juiz de Fora. Synonymy de Family, Genus e espécie. Juiz de Fora, MEC, UFJF. 520p.

Oliveira, M.P.; Vieira, I.; Oliveira, M.H.R. \& Rodrigues, G.J. 1972. Comunicações malacológicas № 7. Contribuição ao conhecimento das sh Brazileiras, litoral do Espirito Santo. Boletim do Instituto de Ciências Biológicas e de Geociências, 4: 1-18.

Oliveira, P.V.; Viana, M.S.S. \& Simone, L.R.L. 2011. Eoholocene malacofauna (Gastropoda, Pulmonata) from a cave of National Park of Ubajara, Ceará state, Brasil. Estudos Geológicos, Recife, 21(1): 85-93.

Oliveira, W.P.; Silva, M.A.V.; Souza, S.A.; Santana, C.C. \& Oliveira-Jr., A.do R. 2017. Caracterização da variabilidade espacial e temporal da precipitação pluviométrica do Centro Sul Baiano. Revista Brasileira de Geografia Física, 10(4): 1143-1151.

Olivier, L. \& Barbosa, F.S. 1955a. Seasonal studies on Australorbis glabratus (Say) from two localities in eastern Pernambuco, Brazil. Publicações Avulsas do Instituto Aggeu Magalhães, 4(6): 79-103.

Olivier, L.\& Barbosa, F.S. 1955b. Seasonal studies on Tropocorbiscentrimentralis in northeastern Brazil. Publicações Avulsas do Instituto Aggeu Magalhães, 4(7): 105-115.

d'Orbigny, A. 1835a. Mollusqués. In: Voyage dans l'Amérique Méridionale. Pitois Levrault, Paris, 5(3): $758+85$ pls.

d'Orbigny, A. 1835b. Synopsis terrestrium et fluviatilum molluscorum, in suo per American Meridionalem itinerere. Magasin de Zoologie, 5(6): 1-44.

Páez, A.P.; Calderón, A.C.; Pineda, I.A.; Velásquez-Trujillo, L.E. \& Ruiz-Sáenz, J. 2014. Un gigante olvidado: llamado a la conservación del caracol nativo Megalobulimus oblongus (Mollusca, Strophocheilidae) en Colombia. Spei Domus, 10(20): 49-51.

Paraense, W.L. 1958. The genera Australorbis, Tropicorbis, Biomphalaria, Platytaphius and Taphius. Revista Brasileira de Biologia, 18(1): 65-80.

Paraense, W.L. 1975. Estado atual da Sistemática dos planorbídeos Brazileiros. Arquivo Museu Nacional, Rio de Janeiro, 55: 105-128.

Paraense, W.L. 1986. Physa Marmorata Guilding, 1828 (Pulmonata: Physidae). Memórias do Instituto Oswaldo Cruz, 81(4): 459-469.

Paraense, W.L. 2006. The Afro-American Biomphalariae. Memórias do Instituto Oswaldo Cruz, 101(1): 21-27.

Paraense, W.L. \& Deslandes, N. 1955. Observation on the morphology of Australorbis glabratus. Memórias do Instituto Oswaldo (ruz, 53(1): $87-103+4 \mathrm{pl}$.

Parkinson, B.; Hemmen, J. \& Groh, K. 1987. Tropical land shells of the world. Wiesbagen, Verlag Christa Hemmen. 279p.

Parodíz, J.J. 1944. Contribuciones al conocimiento de los moluscos terrestres sudamericanos, I. Comunicaciones Zoologicas del Museo de Historia Natural de Montevideo, 1(8): 1-9.

Paula, C.M.; Vaz, A.A.; Vaz, A.A.; Pelizari, G.P.; Robayo, H.M.S.; Garcia, T.D.; Avelino, D.; Zacarin, G.G. \& Smith, W.S. 2017. Ocorrência de um molusco invasor (Melanoides tuberculata, Müller, 1774), em diferentes sistemas aquáticos da bacia hidrográfica do Rio Sorocaba, SP, Brazil. Revista Ambiente \& Água, 12(5): 829-841. 
Paz, R.J.; Watanabe, T.; Dijick, M.P.M. \& Abilio, F.J.P. 1995. First record of Melanoides tuberculata (Müller, 1774) (Gastropoda: Prosobranchia: Thiaridae) in the state of Paraíba (Brasil) and its possible ecological implications. Revista Nordestina de Biologia, 10(2): 79-84.

Pearce, T.A. \& Örstan, A. 2006. Terrestrial Gastropoda. In: Sturm, C.F.; Pierce, T.A. \& Valdés, A. (Eds.). The Mollusks: A guide to their study, collection and preservation. Boca Raton, Universal Publishers. p. 261-285.

Pepe, M.S.; Caldeira, R.L.; Carvalho, 0.S.; Muller, G.; Jannotti-Passos, L.K.; Rodrigues, A.P.; Amaral, H.L. \& Berne, M.E.A. 2009. Biomphalaria molluscs (Gastropoda: Planorbidae) in Rio Grande do Sul, Brazil. Memórias do Instituto Oswaldo Cruz, 104(5): 783-786.

Pfeiffer, L. 1839. Bericht über die Ergebnisse meiner Reisenach Cuba im Winter 1838-1839. Archiv für Naturgeschichte, 5(1): 346-358.

Pfeiffer, L. 1842. Symbolae ad historiam Heliceorum. Cassellis, Sumptibus \& Typis Th. Fisheri. v. 2, 147p.

Pfeiffer, L. 1846. Symbolae ad historiam Heliceorum. Cassellis, Sumptibus \& Typis Th. Fisheri. v. 3, 100p.

Pfeiffer, L. 1848. Monographia Heliceorum Viventium. Lipsiae, F.A. Brockhaus. v. 2, 594p.

Pfeiffer, L. 1853. Monographia Heliceorum Viventium. Lipsiae, F.A. Brockhaus. v. $3,711 p$.

Pfeiffer, L. 1855. Versuch einer Anordnung der Heliceen nach natürlichen gruppen. Malakozoologische Blätter, 2: 112-185.

Pfeiffer, L. 1856. Diagnosen interessanter novitäten. Malakozoologische Blätter, 3: 43-52.

Pfeiffer, L. 1859. Monographia Heliceorum Viventium. Lipsiae, F.A. Brockhaus. v. 4, 919p.

Pfeiffer, L. 1868. Monographia Heliceorum Viventium. Lipsiae, F.A. Brockhaus. v. 6, 598p.

Pieri, O.S.; Raymundo, J.S. \& Jurberg, P. 1980. Estudos sobre o comportamento dos planorbídeos: II - enterramento de Biomphalaria glabrata (Say, 1818) como meio de proteção contra a dessecação. Memórias do Instituto Oswaldo Cruz, 75: 83-88.

Pilate, V.J.; Chicarino, E.D.; Silva, L.C.; Santos, T.V.; Souza, B.A. \& Bessa, E.C.A. 2018. Biologia comportamental comparada entre moluscos terrestres nativos e exóticos. Revista de Biologia Neotropical, 14: 73-81.

Pilsbry, H.A, 1897-1898. Tryon's manual of conchology, second series: pulmonates. Philadelphia, GWDJ. v. 11,339p. +51 pls.

Pilsbry, H.A. 1906. Tryon's manual of conchology, second series: pulmonates. Philadelphia, GWDJ. v. 18, 357p. +51 pls.

Pilsbry, H.A. 1910. Land mollusca of the Panama Canal Zone. Proceedings of the Academy of Natural Sciences of Philadelphia, 62(2): 502-509.

Pilsbry, H.A. 1930. Results of the Pinchot south sea expedition - II. Land mollusks of the Canal Zone The Republic of Panama, and the Cayman Islands. Proceedings of the Academy of Natural Sciences of Philadelphia, 82: 339-354.

Pilsbry, H.A. 1933. Zoological results of the Mato Grosso Expedition to Brazil in 1931 - II Mollusca. Proceedings of the Academy of Natural Sciences of Philadelphia, 85: 67-76.

Pilsbry, H.A. 1934. Review of the Planorbidae of Florida, with notes on other members of the family. Proceedings of the Academy of Natural Sciences of Philadelphia, 86: 29-66.

Pilsbry, H.A. \& Vanatta, E.G. 1905. Hawaiian Species of Endodonta and Opeas. Proceedings of the Academy of Natural Sciences of Philadelphia, 57: 783-786.

Pitoni, V.L.L.; Veitenheimer-Mendes, I.L.; Lanzer, R.M. \& Silva, M.C.P. 1984. Nota sobre a estrutura faunistica do pleuston em um açude no Morro Santana, Porto Alegre, Rio Grande do Sul, Brasil. Ciência e Cultura, 36(2): 245-248.

Pointier, J.P.; David, P. \& Jarne, J. 2005. Biological invasions: the case of planorbid snails. Journal of Helminthology, 79: 249-256.
Porto, R.; Rocha-Filho, J.R.; Johnsson, R. \& Neves, E. 2016. New species of Oxychona (Bulimulidae) from Michelin Ecological Reserve (Bahia state, northeastern Brazil). Journal of (onchology, 42: 105-110.

Potiez, V.L. \& Michaud, A.L.G. 1838. Galérie des Mollusques, ou catalogue méthodique, descriptif et raisonné des mollusques et coquilles du Muséum de Douai. J.B. Paris, Bailliére v. 1, 560p.

Quintana, M.G. 1982. Catálogo preliminar de la malacofauna del Paraguy. Revista del Museo Argentino de Ciencias Naturales "Bernardino Rivadavia" (Zoologia), 11(3): 61-158.

Ramírez, R.; Borda, V.; Romero, P.; Ramirez, J.; Congrains, C.; Chirinos, J.; Ramírez, P.; Velásquez, L.E. \& Mejía, K. 2012. Biodiversidad y endemismo de los caracoles terrestres Megalobulimus y Systrophia en la Amazonia occidental. Revista Peruana de Biología, 19(1):59-74.

Ramírez, R.; Paredes, C. \& Arenas, J. 2003. Moluscos del Perú. Revista de Biología Tropical, 51(3): 225-284.

Raut, S.K. \& Barker, G.M. 2002. Achatina fulica Bowdich and other Achatinidae as pests in tropical agriculture. In: Barker, G.M. (Ed.). Molluscs as Crop Pests. Wallingford, CABI Publishing. p. 55-114.

Reeve, L.A. 1849a. Monograph of the genus Achatina. In: Reeve, Conchologia Iconica. London, Behan. v. 5, 23p.

Reeve, L.A. 1849b. Monograph of the genus Bulimus. In: Reeve, Conchologia Iconica. London, Behan. v. 5, 89p.

Régnier, C.; Fontaine, B. \& Bouchet, P. 2009. Not knowing, not recording, not listing: numerous unnoticed mollusk extinctions. Conservation Biology, 23: 1214-1221.

Richards, H.G. \& Hummelinck, P.W. 1940. Land and freshwater mollusks from Magarita Island, Venezuela. Notula Naturae, 62: 1-16.

Richardson, C.L. 1995. Bulimulidae: catalog of species. Tryonia, 28: 1-458.

Robinson, D.G.; Hovestadt, A.; Fields, A. \& Breure, A.S.H. 2009. The land Mollusca of Dominica (Lesser Antilles), with notes on some enigmatic or rare species. Zoologische Mededelingen, 83(3): 615-650.

Rocha-Miranda, F. \& Martins-Silva, M.J. 2006. First record of the invasive snail Melanoides tuberculatus (Gastropoda: Prosobranchia: Thiaridae) in the Paranã River basin, G0, Brazil. Brazilian Journal of Medical and Biological Research, 66(4): 1109-1115.

Roldán, E.J.; López Martínez, J.; Ramírez, R. \& Trujillo, L.E.V. 2014. Análisis morfológico del sistema reproductor e identificación molecular a través de los marcadores mitocondriales COI y 165 rRNA de Megalobulimus oblongus (Mollusca, Strophocheilidae) de Colombia. Revista Peruana de Biología, 21(1): 79-88.

Rosa, E. 1987. Observações ecológicas sobre Biomphalaria straminea (Dunker, 1848) em áreas do Nordeste, Brasil. Memórias do Instituto Oswaldo Cruz, 82(4): 311-314.

Rueda, A.; Caballero, R.; Kaminsky, R. \& Andrews, K.L. 2002. Vaginulidae in Central America, with emphasis on the bean slug Sarasinula plebeia (Fischer). In: Barker G.M. (Ed.). Molluscs as crop pests. CABI Publishing. p. 115-144.

Salgado, N.C. \& Coelho, A.C.S. 2003. Moluscos terrestres do Brazil (gastrópodos operculados ou não, exclusive Veronicellidae, Milacidae e Limacidae). Revista de Biología Tropical, 51(suppl. 3): 149-189.

Salvador, R.B. 2018. Notes on a new collection of Streptaxidae (Gastropoda: Stylommatophora) from Brazil, with descriptions of two new species of Streptaxis. Integrative Systematics, 1: 25-33.

Salvador, R.B. 2019. Land snail diversity in Brazil. Strombus, 25: 10-20.

Salvador, R.B. \& Cavallari, D.C. 2012. A new Oxychona species (Gastropoda: Pulmonata: Orthalicidae) from Bahia state, Brazil. Journal of Conchology, 41(3): 315-318.

Salvador, R.B. \& Cavallari, D.C. 2014. A new species of Leiostracus from Bahia, Brazil (Gastropoda, Pulmonata, Orthalicidae). Iheringia. Série Zoologia, 104(1): 39-42. 
Salvador, R.B. \& Simone, L.R.L. 2015. Taxonomical study on a sample of land snails from Alcobaça (Bahia, Brazil), with description of a new species. Stuttgarter Beiträge zur Naturkunde A, Neue Serie, 8: 1-7.

Salvador, R.B. \& Simone, L.R.L. 2016. A new species of Kora from Bahia, Brazil (Gastropoda: Pulmonata: Orthalicoidea), with an emended diagnosis of the genus. Stuttgarter Beiträge zur Naturkunde A, Neue Serie, 9: 1-7.

Salvador, R.B.; Cavallari, D.C. \& Simone, L.R.L. 2015. Taxonomical study on a sample of land snails from southeastern Tocantins state, Brazil, with description of a new species. Journal of Conchology, 42(1): 67-78.

Salvador, R.B.; Cavallari, D.C. \& Simone, L.R.L. 2017. Taxonomical study on a sample of land and freshwater snails from caves in central Brazil, with description of a new species. Zoosystematics and Evolution, 93(1): 135-141.

Salvador, R.B.; Charles, L.; Simone, L.R.L. \& Maestrati, P. 2018. Terrestrial gastropods from Pedra Talhada Biological Reserve, Alagoas state, Brazil, with description of a new species of Radiodiscus (Gastropoda: (haropidae). Archiv für Molluskenkunde, 147(1): 101-128.

Salvador, R.B.; Lima, M.S.; Lima, W.C.; Simone, L.R.L. \& Guilherme, E. 2020. First report of Helicina chionea Pilsbry, 1949 from Brazil (Gastropoda, Helicinidae) and new records of Helicina spp. from Acre. Check List, 16(1): 63-66.

Salvador, R.B.; Silva, F.S.; Cavallari, D.C.; Simone, L.R.L. 2021. Terrestrial Gastropoda from the caves of Presidente Olegário, southeastern Brazil. Biota Neotropica, 21(2): e20201169.

Santos, C.M. \& Eskinazi-Sant'Anna, E.M. 2010. The introduced snail Melanoides tuberculatus (Muller, 1774) (Mollusca: Thiaridae) in aquatic ecosystems of the Brazilian Semiarid Northeast (Piranhas-Assu River basin, State of Rio Grande do Norte). Brazilian Journal of Medical and Biological Research, 70(1): 1-7.

Santos, S.B. \& Monteiro, D.P. 2001. Composição de gastrópodes terrestres em duas áreas do Centro de Estudos Ambientais e Desenvolvimento Sustentado (CEADS), Vila Dois Rios, Ilha Grande, Rio de Janeiro, Brazil um estudo-piloto. Revista Brasileira de Zoologia, 18(1): 181-190.

Santos, S.B.; Rodrigues, C.L.; Nunes, G.K.M.; Miyahira, I.C.; Viana, T.A.; Oliveira, J.L.; Fonseca, F.C. \& Silva, P.S.C. 2010. Estado do conhecimento da fauna de invertebrados não marinhos da Ilha Grande (Angra dos Reis, RJ). Oecologia Australis, 14(2): 504-549.

Sá-Oliveira, J.C.; Araújo, F.L.; Teixeira-Filho, R.G.; dos Santos, W.S. \& Ferrari, S.F. 2016. Education as Controlling Factor of Invasive Species (Achatina fulica) in an Amazonian City, Brazil. Creative Education, 7: 159-170.

Sawaya, J. \& Peterson, J.A. 1962. Sôbre a ocorrência de Strophocheilidae (Molusco gastrópode) no Rio Grande do Sul. Boletim da Faculdade de Filosofia, Ciências e Letras, Universidade de São Paulo. Zoologia, 24(24): 31-39.

Say, T. 1818. Account of two new genera, and several new species, of fresh water and land shells. Journal of the Academy of Natural Sciences of Philadelphia, 1: 276-284.

Scarabino, F. 2003. Lista sistemática de los Gastropoda terrestres vivientes de Uruguay. Comunicaciones de la Sociedad Malacológica del Uruguay, 8(78-79): 203-214.

Schalie, H. 1940. Notes on mollusca from Alta Vera Paz, Guatemala. Occasional Papers of the Museum of Zoology University of Michigan, 413: 1-11.

Scott, M.I.H. 1957. Estudio morfologico y taxonomico de los Ampullariidos de la Republica Argentina. Revista del Museo Argentine de Ciencias Naturales "Bernardino Rivadavia", Ciencias Zoologicas, 3(5): 231-333.

Shade, F.H. 1965. Lista de moluscos del Guaira (Villarrica-Paraguay) conocidos hasta el presente. Comunicaciones de la Sociedad Malacológica del Uruguay, 1(8): 209-221.

Silva, C.C. \& Castro, G.A. 2003. Gastrópodes terrestres num fragmento de restinga no estado do Espírito Santo. Bioikos, 17(1/2): 65-69.
Silva, E.C. \& Aleluia, F.T.F. 2010. Ocorrência de Achatina fulica Bowdich, 1822 (Mollusca, Gastropoda) em Salvador, Bahia, Brazil. Revista Brasileira de Zoociências, 12(2): 199-204.

Silva, E.C. \& Gomes, L.E.0. 2014. Melanoides tuberculatus (Müller, 1774): Occurrence extension of the invasive gastropod in Bahia, Brazil. PanAmerican Journal of Aquatic Sciences, 9(2): 145-149.

Silva, E.C. \& Omena, E.P. 2014. Population dynamics and reproductive biology of Achatina fulica Bowdich, 1822 (Mollusca, Gastropoda) in Salvador Bahia. Biota Neotropica, 14(3): 1-11.

Silva, F.S.; Cavallari, D.C. \& Simone, L.R.L. 2019a. New records of Helicina schereri (Gastropoda: Helicinidae) from the states of Bahia, Minas Gerais, and Mato Grosso do Sul, Brazil. Papéis Avulsos de Zoologia, 59: 2-4.

Silva, F.S.; Simone, L.R., \& Salvador, R.B. 2019b. Taxonomic study on a collection of terrestrial mollusks from the region of Santa Maria, Rio Grande do Sul state, Brazil. Arquivos de Zoologia, São Paulo, 50(3): 175-190.

Silva, J.M.C.; Tabarelli, M.; Fonseca, M.T. \& Lins, L.V. 2003. Biodiversidade da caatinga: áreas e ações prioritárias para a conservação. Brasília, Ministério do Meio Ambiente, Universidade Federal de Pernambuco. 382p.

Silva, R.E.; Melo, A.L.; Pereira, L.A. \& Frederico, L.F. 1994. Levantamento malacológico da bacia hidrográfica do lago Soledade, Oura Branco (Minas Gerais, Brasil). Revista do Instituto de Medicina Tropical de São Paulo, 36(5): 437-444.

Simone, L.R.L. 1999. Mollusca Terrestres. In: Brandão, C.R. \& Cancello, E.M. (Eds.). Biodiversidade do Estado de São Paulo, Brazil: síntese do conhecimento ao final do século XX. FAPESP, São Paulo, 5: 3-8.

Simone, L.R.L. 2001. Phylogenetic analyses of Cerithioidea (Mollusca, (aenogastropoda) based on comparative morphology. Arquivos de Zoologia, São Paulo, 36(2): 147-263.

Simone, L.R.L. 2004. Comparative morphology and phylogeny of representatives of the superfamilies of Architaenioglossans and the Annulariidae (Mollusca, Caenogastropoda). Arquivos do Museu Nacional, Rio de janeiro, 62(4): 387-504.

Simone, L.R.L. 2006. Land and freshwater mollusks of Brazil. São Paulo, Editora Gráfica Bernardi \& Fundação de Amparo à Pesquisas do Estado de São Paulo. 390p.

Simone, L.R.L. 2012. Taxonomical study on a sample of pulmonates from Santa Maria da Vitória, Bahia, Brazil, with description of a new genus and four new species (Mollusca: Orthalicidae and Megalobulimidae). Papéis Avulsos de Zoologia, 52(36): 431-439.

Simone, L.R.L. \& Casati, R. 2013. New land mollusk fauna from Serra da Capivara, Piauí, Brazil, with a new genus and five new species (Gastropoda: Orthalicoidea, Streptaxidae, Subulinidae). Zootaxa, 3683(2): 145-158.

Simone, L.R.L. \& Salvador, R.B. 2021. A new species of Spiripockia from eastern Brazil and reassignment to Cochliopidae (Gastropoda, Truncatelloidea). Journal of Natural History, 54(47-48): 3121-3130.

Smith, E.A. 1895. Report on the land and freshwater shells collected by Mr. Herbert H. Smith at St. Vincent, Grenada and other neighbouring islands. Proceedings of the Malacological Society of London, 1: 300-325.

Soares, A.A.; Thiengo, S.C.; Cadei, M. de Sá; Locks, M.; Beltrão, M. \& Fernandez, M.A. Non-formal education for schistosomiasis prevention: The experience of the Museu Arqueológico de Central, Bahia, Brazil. Memórias do Instituto Oswaldo Cruz, 101(1): 107-10

Sodeman-Jr., W.A. 1973. The influence of light on Biomphalaria glabrata. Nautilus, 87(4): 103-106.

Solem, A. 1956. Non-marine Mollusca from Salobra, Matto Grosso, Brazil and a collection of South Brazilian Artemon. Notulae Naturae, 287: 1-14.

Souza, C.P.; Caldeira, R.L.; Drummond, S.C.; Melo, A.L.; Guimarães, C.T.; Soares, D.M. \& Carvalho, O.S. 2001. Geographical distribution of Biomphalaria 
snails in the state of Minas Gerais, Brazil. Memórias do Instito Oswaldo Cruz, 96(3): 293-302.

Souza, C.P.; Lima, L.C.; Jannotti-Passos, L.K.; Ferreira, S.S.; Guimarães, C.T.; Vieira, I.B.F. \& Mariani-Júnior, R. 1998. Moluscos límnicos da microregião de Belo Horizonte, MG, com ênfase nos vetores de parasitoses. Revista da Sociedade Brasileira de Medicina Tropical, 31: 449-456.

Souza, C.P.; Ribeiro, P.R.B.; Guimarães, C.T.; Jannotti-Passos, L.K. \& Souza, M.A. 1996. Esquistossomose: nova ocorrência de Biomphalaria straminea em Belo Horizonte, Minas Gerais. Cadernos de Saúde Pública, 12(4):541-544.

Sowerby, G.B. 1820. The genera of recente and fóssil shells, for the use of students in conchology and Geology. London, G.B. Sowerby. 275p.

Spix, J.A. 1827. Testacea Fluviatilia quae in itinere per Brazilian. Monachii, Typis C. Wolf. $36 p+28$ pls.

Starmühler, F. 1969. Die gastropoden der Madagassischen Binnengewässer. Malacologia, 8(1-2): 1-434.

Strong, E.E.; Colgan, D.J.; Healy, J.M.; Lydeard, C.; Ponder, W.F. \& Glaubrecht, M. 2011. Phylogeny of the gastropod superfamily Cerithioidea using morphology and molecules. Zoological Journal of the Linnean Society, 162: 43-89.

Taylor, D.W. 2003. Introduction to Physidae (Gastropoda: Hygrophila); biogeography, classification, morphology. Revista de Biología Tropical, 51(1): 1-287

Teixeira, D.G.; Guerra, J.L.; Silva, Z.; Azarias, R.E.G.R. \& Martins, M.F. 2008. Aspectos macroscópicos da anatomia do sistema reprodutor de escargots: Achatina fulica e Achatina monochromatica. Brazilian Journal of Veterinary Research and Animal Science, 45(5): 333-338.

Teles, H.M.S. 1996. Distribution de Biomphalaria straminea do Sul da Região Neotropical, Brazil. Revista de Saúde Pública, 30(4): 341-349.

Teles, H.M.S. \& Fontes, L.R. 2002. Implicações da introdução e dispersão de Achatina fulica. Boletim do Instituto Adolfo Lutz, 12(1): 3-5.

Teles, H.M.S. \& Vaz, J.F. 1988. Distribution de Biomphalaria straminea (Dunker, 1848) (Pulmonata, Planorbidae) no estado de São Paulo, Brazil. Ciência e Cultura, 40(2): 173-176.

Teles, H.M.S.; Pereira, P.A.C. \& Richinitti, L.M.Z. 1991. Distribution de Biomphalaria (Gastropoda, Planorbidae) nos Estados do Rio Grande do Sul e Santa Catarina, Brazil. Revista de Saúde Pública, 25(5): 350-352.

Teles, H.M.S.; Vaz, J.F.; Fontes, L.R. \& Domingos, M.F. 1997. Registro de Achatina fulica Bowdich, 1822 (Mollusca, Gastropoda) no Brazil: caramujo hospedeiro intermediário da angiostrongilíase. Revista de Saúde Pública, 31(3): 310-312.

Thiengo, S.C.; Faraco, F.A.; Salgado, N.C.; Cowie, R.H. \& Fernandez, M.A. 2007. Rapid spread of an invasive snail in South America: The giant African snail, Achatina fulica, in Brazil. Biological Invasions, 9: 693-702.

Thiengo, S.C.; Fernandez, M.A.; Boaventura, M.F. \& Stortti, M.A. 1998. A Survey of Freshwater Gastropods in the Microrregião Serrana of the State of Rio de Janeiro, Brazil. Memórias do Instituto Oswaldo Cruz, 93(suppl. 1): 233-234.

Thiengo, S.C.; Santos, S.B. \& Fernandez, M.A. 2005. Malacofauna límnica da área de influência do lado da usina hidrelétrica de Serra da Mesa, Goiás, Brasil. Estudo qualitativo. Revista Brasileira de Zoologia, 22(4): 867-874.
Thomé, J.W. 1969. Redescrição dos tipos de Veronicellidae (Mollusca, Gastropoda) Neotropicais: I. Espécies depositadas no "Zoologisches Museum" de Kiel, Alemanha. Iheringia, 37: 101-111.

Thomé, J.W. 1975. Estado atual da sistemática dos Veronicellidae Americanos. Arquivos do Museu Nacional, Rio de Janeiro, 55: 155-165.

Thomé, J.W. 1988. Lista das Veronicellidae (Mollusca; Gastropoda) da Jamaica. Revista de Biologia Tropical, 48(8): 807-810.

Thomé, J.W. 1993. Estado atual da sistemática dos Veronicellidae (Mollusca: Gastropoda) americanos, com comentários sobre sua importância econômica, ambiental e na saúde. Biociências, Porto Alegre, 1(1): 61-75.

Thomé, J.W. 1994. Annotated listo $f$ the Veronicellidae (Gastropoda: Soleolifera) of Venezuela. Malacological Review, 27: 43-46.

Thomé, J.W.; Gomes, S.R. \& Picanço, J.B. 2006. Os caracóis e as lesmas dos nossos bosques e jardins. Rio Grande do Sul, União Sul-Americana de Estudos da Biodiversidade - USEB. 123p.

Thomé, J.W.; Gomes, S.R. \& Silva, R.S. 1999. Ocorrência e Distribution da Family Veronicellidae Gray, 1840 (Mollusca, Gastropoda) no Rio Grande do Sul, Brazil. Biociências, Porto Alegre, 7(I): 157-165.

Thomé, J.W., Santos, P.H. \& Pedott, D.L. 1997. Annotated list of Veronicellidae from the collections of Academy of Natural Sciences of Philadelphia and National Museum of Natural History, Smithsonian Institution, Washington, D.C., U.S.A. (Mollusca: Gastropoda: Soleolifera). Proceedings of the Biological Society of Washington, 110(4): 520-536.

Thompson, F.G. 2011. An annotated checklist and bibliography of the land and freshwater snails of Mexico and Central America. Bulletin Florida Museum of Natural History, 50(1): 1-299.

Tillier, S. 1980. Gastéropodes terrestres et fluviatiles de Guyane Française. Mémoires du Muséum National d'Histoire Naturelle, Sér. A, Zoologie, 118: 1-189.

Trouy, K.C.; Alencar, A.P.S. \& Silva, J.S. 2016. Levantamento da malacofauna no município de Juína, Mato Grosso, Brazil. Revista de Extensão Universitária, 5: $117-126$

Valim, D.S.\& Bim, S. 2017. Disseminação do caracol gigante africano (Achatina fulica): ameaça ecológica, agrícola e sanitária. Revista Maiêutica, 5(1): 7-15.

Vasconcellos, M.C. \& Pile, E. 2001. Ocorrência de Achatina fulica no Vale do Paraíba, Estado do Rio de Janeiro, Brasil. Revista de Saúde Pública, 35(6): 582-584.

Vidigal, T.H.D.A.; Marques, M.M.G.S.M.; Lima, H.P. \& Barbosa, F.A.R. 2005. Gastrópodes e bivalves límnicos do trecho médio da bacia do Rio Doce, Minas Gerais, Brazil. Lundiana, 6(suppl.): 67-76.

Vieira, P.C. \& Simone, L.R.L. 1990. Malacofauna na gruta da Pescaria, I poranga, SP. Revista do Instituto Geológico, 8-10(1): 57.

Vogler, R.E.; Beltramino, A.A.; Sede, M.M.; Gregoric, D.E.G.; Núñez, V. \& Rumi, A. 2013. The giant African snail, Achatina fulica (Gastropoda: Achatinidae): Using bioclimatic models to identify South American areas susceptible to invasion. American Malacological Bulletin, 31(1): 39-50.

Zanol, J.; Fernandez, M.A.; Oliveira, A.P.M. \& Thiengo, S.C. 2010. 0 caramujo exótico invasor Achatina fulica (Stylommatophora, Mollusca) no Estado do Rio de Janeiro (Brazil): situação atual. Biota Neotropica, 10(3): 447-451. 